\title{
IV. Jüdische Partisanen
}

\section{Einleitung}

Die drittstärkste ethnische Gruppe im Verband Baranoviči stellten jüdische Partisanen dar. Ein Teil von ihnen gehörte den ethnisch gemischten Abteilungen an. Es gab aber auch rein jüdische Gruppen, die im Rahmen der sowjetischen Partisanenbewegung operierten. ${ }^{1}$ In der Oblast' Baranoviči waren sogar die zwei größten rein jüdischen Partisaneneinheiten während des Zweiten Weltkrieges in Europa, die Bielski-Abteilung und die Zorin-Abteilung, aktiv. Hinzu kamen zahlreiche kleinere jüdische „Überlebensgruppen“, deren genau Zahl unbekannt ist und von denen wohl die meisten in den Jahren 1942-1944 vernichtet wurden.

Mit der deutschen Besetzung begann in der Oblast' Baranoviči die systematische Verfolgung der jüdischen Bevölkerung. Bereits im Sommer und Herbst 1941 ermordeten deutsche Sicherheits- und Polizeikräfte Tausende von Juden, wobei vor allem das flache Land "gesäubert" wurde. Im Winter 1941/42 ging das Morden weiter. Das Schlimmste stand aber erst noch bevor. Im Mai 1942 wurde das Gebiet der Oblast' wie auch die übrigen Teile des westlichen Weißrußlands von einer großen Vernichtungswelle erfaßt, die bis Ende 1942 andauerte und der die meisten der dort lebenden Juden zum Opfer fielen. Kommandos der deutschen Sicherheitspolizei und des SD, der deutschen Gendarmerie und Schutzmannschaften, die sich aus einheimischen Kollaborateuren zusammensetzten, und eng mit der deutschen Zivilverwaltung kooperierten, löschten systematisch ganze jüdische Gemeinden aus, in der Regel durch Massenerschießungen. Die Mordkommandos setzten aber auch Gaswagen ein. ${ }^{2}$

Bis Ende 1942 wurden die meisten Juden in der Oblast' Baranoviči ermordet, einige Tausend lebten noch in einzelnen Ghettos als Zwangsarbeiter oder befanden sich auf der Flucht. Denn die mit der totalen Vernichtung konfrontierten Juden leisteten aktiven und passiven Widerstand, und die einzige reale Chance zum Über-

1 Leonid Smilovitsky schätzt, daß sich etwa 15000 Juden den weißrussischen Partisanen angeschlossen haben. Leonid Smilovitsky, „Righteous Gentiles, the Partisans, and Jewish Survival in Belorussia 1941-1944", in: Holocaust and Genocide Studies 11/3 (1997), S. 318; diese Zahl erscheint jedoch zu hoch gegriffen. Die Personalabteilung des CSPD verfügte zum 15. 2. 1944 über Angaben zur ethnischen Herkunft von 80170 Partisanen in Weißrußland. Danach waren 51158 von ihnen Weißrussen, 20086 Russen, 3530 Ukrainer und 5396 anderer Nationalität. Bei den Letzteren waren wohl die meisten jüdischer Herkunft. Insgesamt gab es zu diesem Zeitpunkt etwa 120000 Partisanen in Weißrußland. Vgl. Tätigkeitsbericht der Personalabteilung des CŠPD vom 28. 2. 1944 für die Zeit vom 15. 7. 1942-15. 2. 1944, abgedruckt in: Partizanskoe dviženie v gody Velikoj Otečestvennoj Vojny 1941-1945 gg. Dokumenty i materialy. Moskva 1999, S. 463-486, hier S. 480, 483.

2 Vgl. dazu Gerlach, Kalkulierte Morde, S. 536-554, 609-627, 688-708. 
leben bestand in der Flucht. Viele Juden flohen nun aus den Ghettos in die umliegenden Wälder und versuchten dort zu überleben. Die Besatzungskräfte starteten jedoch sogleich eine Hetzjagd auf sie. Der Gendarmerie-Oblastführer in Baranoviči meldete am 26. August 1942 an seinen Vorgesetzten in Minsk:

Vom Oblastkommissar in Baranowitsche habe ich allgemein den Auftrag, soweit es meine Kräfte zulassen, das Gebiet, insbesondere das flache Land, von Juden zu befreien. Die in den letzten Monaten durchgeführten größeren Aktionen hatten zur Folge, daß in sehr starkem Maße Juden geflüchtet und zu den Banditengruppen übergetreten sind. Um einer weiteren Flucht vorzubeugen, habe ich die in den Orten Polonka und Mir noch vorhandenen Juden beseitigt. Insgesamt wurden 719 erschossen. Inzwischen konnten von den Gend. - Posten bereits 320 Juden, die bei den größeren Aktionen geflüchtet waren, wieder erfaßt und standrechtlich erschossen werden. ${ }^{3}$

Tausende von Juden wurden in den Ghettos unter dem grotesken, Ursachen und Folgen verkehrenden Vorwand ermordet, ihrer Flucht vorbeugen zu wollen. Ein Großteil derjenigen, die tatsächlich versuchten, den Mordkommandos zu entkommen, kam bald ums Leben. Die anderen schlossen sich zu "Überlebensgruppen“ zusammen und versuchten, sich unter den äußerst widrigen Verhältnissen zu behaupten. Hunderte, die mehr Glück hatten, konnten sich den sowjetischen Partisanen anschließen. In der im Sommer 1944 aufgezeichneten Entstehungsgeschichte der Partisanenbrigade „Leninscher Komsomol“ heißt es:

Im April und Mai 1942, als die Massenmorde an den Juden in den Ghettos begannen, kamen noch Hunderte Juden in den Wald, die vor den deutschen Henkern geflohen waren. In der $\mathrm{Na}-$ činskaja-Pušča, im Wald von Dubiči und in anderen Wäldern bewaffneten und organisierten sich Judengruppen, sie beschlossen, gegen die Okkupanten mit der Waffe in der Hand zu kämpfen. Der Sommer 1942 war die Zeit der Aufstellung dieser Gruppen. ${ }^{4}$

In den Reihen der polnischen Heimatarmee gab es dagegen nur wenige Partisanen jüdischer Herkunft. Es handelte sich dabei hauptsächlich um polnisch assimilierte Intelligenz. Im Jahre 1942, als die große Flucht aus den Ghettos begann, gab es nur wenige Partisanenabteilungen der AK ${ }^{5}$, die Ghettoflüchtlinge aufnehmen konnten. Darüber hinaus begegneten die AK-Einheiten jüdischen Flüchtlingen mit Misstrauen, da den Juden oft pauschal antipolnische und prosowjetische Einstellung vorgeworfen wurde. Aus diesen Gründen kam die Heimatarmee für die jüdischen Ghettoflüchtlinge kaum als eine Alternative zu den sowjetischen Partisanen in Frage ${ }^{6}$, zumal es seitens einzelner AK-Abteilungen auch zu antisemitisch motivierten Übergriffen gekommen war (siehe Dok. 4 und 5).

Die größte jüdische Partisaneneinheit, die während des Zweiten Weltkrieges in der Oblast' Baranoviči als auch in Europa entstand, war die sogenannte Bielski-Abteilung, die im Wald und in der Gegend um Naliboki operierte. Sie war eine „Familiengruppe“: Ein Großteil ihrer Angehörigen, mehr als drei Viertel, war unbewaff-

3 Gend.-Hauptmannschaft in Baranoviče an den Kommandeur der Gendarmerie Weißruthenien, Baranoviče, den 26. 8. 1942: AAN, F 76 (ohne Paginierung).

4 Siehe Kapitel I., Dokument 15, S. 79.

5 Siehe Kapitel V.

6 Kazimierz Krajewski, Der Bezirk Nowogródek der Heimatarmee. Nationalitätenkonflikte und politische Verhältnisse 1939 bis 1945, in: Bernhard Chiari (Hrsg.), Die polnische Heimatarmee. Geschichte und Myhtos der Armija Krajowa seit dem Zweiten Weltkrieg. München 2003, S. $568 \mathrm{f}$. 
net (Frauen, Kinder, Alte). ${ }^{7}$ Nach dem Stand vom 1. April 1944 zählte die BielskiAbteilung 955 Mitglieder, davon 388 Frauen, 26 Kinder bis 7 und 98 zwischen 7 und 16 Jahren alt sowie 167 Personen über 40 Jahre. Fast alle (953) waren Ghettoflüchtlinge. Die bewaffneten Angehörigen der Abteilung hatten die Aufgabe, die Gruppe zu schützen und die Versorgung zu sichern. ${ }^{8}$

Die Bielski-Gruppe entstand im Frühjahr 1942 und setzte sich ursprünglich aus etwa 20 Personen zusammen: Anatolij Bielski, seinen drei Brüder sowie seinen Verwandten und Bekannten. Mit der Zeit wuchs die Gruppe zu einer großen Abteilung, die etwa 1200 Angehörige zählte. ${ }^{9}$ Zunächst operierte sie selbständig. Im September 1942 nahm sie Kontakt mit der Partisanenabteilung des Leutnants V. I. Pančenkov auf. Gemeinsam führten sie danach mehrere Aktionen durch. ${ }^{10} \mathrm{Im}$ Frühjahr 1943 wurde die Bielski Gruppe als die Zweite Kompanie der Abteilung „Oktober“ der Brigade „Kirov“11 in den Partisanenverband Baranoviči eingegliedert. Der Kompanieführer war Anatolij/Tuvia Bielski. ${ }^{12}$ Im Juni 1943 nannte sich die BielskiGruppe Abteilung „Ordžonikidze“ der Brigade „Kirov“.13 Im Herbst 1943 (Ende Oktober/Anfang November) wurde Bielski als Kommandeur der Abteilung „Ordžonikidze“ abgesetzt und zum Kommandeur der „Familiengruppe“ bestimmt, die aus der Abteilung „Ordžonikidze“ ausgegliedert wurde. Die Gruppe unterstand organisatorisch aber weiterhin der Abteilung „Ordžonikidze“.14

Die Familiengruppe Bielski zählte im November 1943 etwa 800 Personen und hielt sich in drei gesonderten Lagern auf: im Winterlager, Kartoffellager und Sommerlager. Außerdem erhielt Bielski vom Vereinigten Rajon-Zentrum Ivenec den Auftrag, alle Familiengruppen, etwa 100 Personen verteilt auf 6 oder 8 Gruppen, die sich in Wäldern in der Gegend um Mir und Stolpcy aufhielten, zu übernehmen. ${ }^{15}$

Das Leben in der Abteilung war strikt geregelt und jeder hatte seine Aufgaben zu

7 Nehama Tec, Defiance. The Bielski Partisans, New York/Oxford 1993, die deutsche Ausgabe erschien unter dem Titel: Bewaffneter Widerstand. Jüdische Partisanen im Zweiten Weltkrieg, Gerlingen 1996, S. 217.

8 Zusammensetzung der Bielski-Partisanenabteilung nach dem Stand vom 1. 4. 1944: NARB, f. 3623, op. 1, d. 4, 1. 21; siehe Kap. IV., Dokument 14.

9 Tec, Bewaffneter Widerstand, S. 74-87; im Bericht vom Frühjahr 1943 behauptete Anatolij/ Tuvia Bielski, seine Gruppe sei im Juli 1941 entstanden. Vgl. Bericht über bewaffnete Aktivitäten der zweiten Kompanie der Abteilung „Oktober“ für die Zeit vom Juli 1941 bis Frühjahr 1943 (ohne Datum, Früjahr 1943): NARB, f. 3500, op. 4, d. 251,1.63. Tatsächlich hielten sich Bielski und seine Brüder an verschiedenen Orten versteckt und schlossen sich erst im Mai 1942 zu einer Gruppe zusammen, wie aus ihren Nachkriegsaussagen hervorgeht. Siehe Tec, Bewaffnter Widerstand, S. 74-87, und Peter Duffy, The Bielski Brothers, New York 2003, S. $55-76$.

10 Bericht über bewaffnete Aktivitäten der zweiten Kompanie der Abteilung „Oktober“ für die Zeit vom Juli 1941 bis Frühjahr 1943 (ohne Datum, Früjahr 1943): NARB, f. 3500, op. 4, d. $251,1.63$.

11 Die Brigade „Kirov“ hieß ursprünglich Briagde „Lenin“. Duffy, Bielski Brothers, S. 169.

12 Bericht über bewaffnete Aktivitäten der zweiten Kompanie der Abteilung "Oktober" für die Zeit vom Juli 1941 bis Frühjahr 1943 (ohne Datum, Früjahr 1943): NARB, f. 3500, op. 4, d. 251,1.63.

13 Befehl des Bevollmächtigten des ZK der KP(b)B und BŠPD im Vereinigten Rajonzentrum Šcučin, Šupienia, vom 7. 6. 1943: NARB, f. 1329, op. 1, d. 82, 1. 2.

14 Bielski an "Platon", 12.11. 1943: NARB, f. 1329, op. 1, d. 45, 1. 17.

15 Bericht des Kommandeurs der Familiengruppe A. Bielski Nr. 0011, ohne Datum, November 1943: NARB, f. 3618, op. 1, d. 6, 1. 129. 
erfüllen. Etwa 100 Angehörige der Familiengruppe arbeiteten in verschiedenen Waldwerkstätten so u.a. in der Bäckerei, Schneiderei, Schuhmacherei, Waffenschmiede oder Metzgerei. Drei Gruppen mit zusammen etwa 60 Angehörigen führten die sogenannten wirtschaftlichen Operationen in verschiedenen Rajons durch, um die Versorgung sicherzustellen. Anfang Dezember 1943 waren u.a. folgende Wintervorräte angelegt: $200 \mathrm{t}$ Kartoffeln, $3 \mathrm{t}$ Kohl, $5 \mathrm{t}$ Rüben, $5 \mathrm{t}$ Getreide sowie cirka $3 \mathrm{t}$ Fleisch und $1 \mathrm{t}$ Wurst. Zum Schutz der Gruppe standen 66 Partisanen mit einem Aufklärungstrupp von 6 Mann zur Verfügung. Sonstige bewaffnete Aktionen führten die Partisanen der Gruppe zu diesem Zeitpunkt nicht durch. ${ }^{16}$

Am 3. Januar 1944 gliederte „Platon“ per Befehl die Familiengruppe Bielski aus der Brigade "Kirov“ aus und bildete die selbständige Abteilung Bielski, die ihm direkt unterstellt war. Zugleich erhielt Anatolij Bielski, den „Platon“ zum Kommandeur der Abteilung ernannte, den Befehl, eine Kampfgruppe aufzustellen, die bewaffnete Einsätze durchzuführen hatte. ${ }^{17}$ Am 1. Februar 1944 stellte Bielski eine solche Kampfgruppe mit 180 Partisanen auf. ${ }^{18}$ Am 6. Mai 1944 ließ „Platon“ die Bielski-Abteilung noch einmal umbenennen, diesmal in „M. I. Kalinin“.19 Zwei Monate später (am 12. Juli 1944), nachdem die Truppen der Roten Armee das Gebiet befreit hatten, löste Anatolij Bielski die Abteilung „Kalinin“ auf.20

Auch die oben erwähnte Zorin-Abteilung war eine „Familiengruppe“. Sie bestand offiziell als Abteilung Nr. 106 des Vereinigten Rajon-Zentrums Ivenec, deren Kommandeur Siemon Zorin war. Er erhielt den Auftrag, mit 40 jüdischen Partisanen eine Partisanenabteilung zu bilden, sie weiter aufzubauen und sowohl diejenigen Juden zu retten, die sich in den Wäldern aufhielten, als auch die, welche erst dorthin kommen sollten. ${ }^{21}$ Zum 1. Oktober 1943 zählte die Abteilung etwa 700 Angehörige, von denen 450 bewaffnet waren. ${ }^{22}$

Darüber hinaus bestanden auf dem Territorium der Oblast' Baranoviči zahlreiche kleinere jüdische Familien- und Überlebensgruppen, die lose mit den sowjetischen Partisanen in Kontakt standen oder aber selbständig operierten. Sie setzten sich, wie die meisten übrigen jüdischen Partisanen auch, aus den Ghettoflüchtlingen zusammen. Diese Gruppen führten in der Regel keine militärischen Operationen gegen den Feind durch, sondern konzentrierten sich auf das Überleben in den Wäldern. Sie waren durch deutsche Aktionen, die gegen Partisanen gerichtet waren, besonders gefährdet, da sie klein und oft schlecht bewaffnet waren. Aber auch marodierende Partisanen und gewöhnliche kriminelle Banden stellten für sie eine Bedrohung dar, da sie als leichte und zugleich lohnende Beute galten. ${ }^{23}$

16 Tätigkeitsbericht der Führung der Familiengruppe der Abteilung „Ordžonikidze“ vom 5. 12. 1943: NARB, f. 3623, op. 1, d. 2, 11. $95 \mathrm{f}$.

17 Befehl Nr. 001 des Bevollmächtigten des CŠPD und des ZK der KP(b)B für die Oblast' Baranoviči vom 3. 1. 1944: NARB, f. 3423, op. 1, d. 1, l. 1.

18 Befehl Nr. 6 vom 1. Febraur 1944: NARB, f. 3623, op. 1, d. 3.

19 Befehl Nr. 0033 vom 6. 5. 1944, Kapitel IV., S. 216.

20 Siehe den Befehl Nr. 0041 vom 12. 7. 1944, Kapitel IV.

21 Anatol Wertheim, Zydowska Partyzantka na Białorusi, in: Zeszyty Historyczne, 1988 (Hcft 86), S. 96-162, hier S. 142.

22 Bericht über Stand des Untergrundes und der Partisanenbewegung in der Oblast' Baranoviči zum 1. 10. 1943, unvollständige Angaben: NARB, f. 1329, op. 1, d. 8, 1l. 1-9, 15.

$23 \mathrm{Vgl}$. Tec, Bewaffneter Widerstand, S. $117 \mathrm{f}$. 
Das Verhältnis der sowjetischen nichtjüdischen Partisanen zu den jüdischen Partisanen und Flüchtlingen war zwiespältig. Es kam nicht selten zu Konflikten zwischen einzelnen Partisanen und sogar ganzen Gruppen, die auch Todesopfer forderten.

Manche Flüchtlinge lebten in panischer Angst vor den sowjetischen Partisanen. Anatol Wertheim flüchtete im Herbst 1942 mit seinen Freunden aus dem Ghetto Stolbcy und überwinterte im Wald in einem Erdbunker. Ihr Ziel war es, sich den sowjetischen Partisanen anzuschließen. Im Frühjahr 1943 begegneten sie im Wald zwei anderen jüdischen Flüchtlingen, die sie nach Partisanen befragten. Ihre Antwort war: „Partisanen? Sie sind ja schlimmer als die Deutschen!“ - und sie begannen zu erzählen über Morde, Vergewaltigungen und Raubüberfälle, die Partisanen an den sich in den Wäldern versteckten Juden begingen. ${ }^{24}$ Nechama Tec schreibt dazu:

Jüdische Flüchtlinge wurden zu einer leichten Beute. Sie waren nicht nur den deutschen Übergriffen ausgeliefert, sondern auch vom guten Willen anderer, für das Leben im Freien besser ausgerüsteter Waldbewohner abhängig. Häufig begegneten den gerade entronnenen Juden in den Wäldern neue Formen von Verfolgung. Manche wurden beraubt und mißhandelt oder sogar ermordet. Die Raubüberfälle wurden in der Regel aus reiner Habgier begangen; Antisemitismus spielte als Motiv kaum eine Rolle. In den meisten Fällen wurde allerdings die Erwartung auf reiche Beute enttäuscht. ${ }^{25}$

Für die jüdischen Flüchtlinge war die Situation in den Wäldern undurchschaubar. Einige sowjetische Partisaneneinheiten wiesen sie ab und verfolgten sie sogar manchmal, andere wiederum übernahmen sie oder halfen ihnen. So erteilte beispielsweise der Kommandeur der Brigade „Pervomajskaja“ seinen Partisanen den Befehl, allen jüdischen Familien, die sich im Operationsraum der Brigade versteckt hielten, zu helfen. ${ }^{26}$ Wie bereits ausgeführt, wurde die Zorin-Abteilung speziell dazu gebildet, jüdischen Flüchtlingen Schutz zu bieten.

In zeitgenössischen polnischen Berichten wird oft beklagt, daß jüdische Partisanen bzw. ,jüdische Banden“ besonders brutal gegen die nichtjüdische Zivilbevölkerung vorgingen. Im März 1943 meldete die Abteilung „Korweta“ der Heimatarmee in der Region Nowogródek ,häufige Raubüberfälle, besonders jüdischer Banden, die sehr grausam und rücksichtslos sind". ${ }^{27}$ Im Mai und Juni 1943 liefen Verhandlungen zwischen der Führung der Heimatarmee im Bezirk Nowogródek und der Führung der sowjetischen Partisanenbrigade "Lenin“. Eine der Forderungen, die der polnische Vertreter am 8. Juni 1943 vorbrachte, war: „Nichtentsendung von Juden zu Requirierungen. Die Bevölkerung beklagt sich darüber, sie greift spontan zu Waffen, um sich zu wehren, weil jene brutal sind, vergewaltigen Frauen und kleine Kinder, drohen den Älteren mit späterer Rache der Sowjets, sie kennen keine Grenzen in ihrer unbegründeten Bösartigkeit und Raubgier". ${ }^{28}$ Die Gemeindeverwal-

24 Wertheim, Żydowska Partyzantka S. 136.

$25 \mathrm{Tec}$, Bewaffneter Widerstand, S. 115.

26 Meldung des Kommandeurs der Brigade „Pervomajskaja“, Hauptmann Kovalev an den Bevollmächtigten des ZK der $\mathrm{KP}(\mathrm{b}) \mathrm{B}$ und den Vertreter des Generalstabes der Roten Armee vom Juni 1943: NARB, f. 3500, op. 4, d. $250,1.8$.

27 „Korweta“ Bericht über den Stand „K“ für die Zeit vom 20. Febraur bis zum 20. 3. 1943: AAN, MF 423, Bl. 356.

28 Protokoll über die Gespräche zwischen dem Delegierten des Hauptstabes der polnischen Partisanen "Ost" und der Führung der Partisanenbrigade "Lenin" vom 8.6. 1943, abgedruckt in: Studie Podlaskie (VI) 1996, S. 231-234, hier S. 232. 
tung Niedzwiedzica forderte im Sommer 1942 sofortige Hilfe von der Gendarmerie-Führung in Baranoviči: „Die Gemeindeverwaltung meldet, daß die Banditen (meistens Juden) jede Nacht in den Dörfern unserer Gemeinde rauben und plündern. Die Bevölkerung ist nur noch mit Unterhosen und Blusen bekleidet, da die Banditen alles weggenommen haben. [...] Es wird um sofortige Hilfe gegen diese Banden (Juden) gebeten." 29

Es ist heute kaum möglich, all diese Vorwürfe zu verifizieren; die in den Formulierungen der Berichte häufig zutage tretenden antisemitischen Einstellungen und Wahrnehmungsmuster sowie die Faktizität der beklagten Ereignisse müssen gesondert betrachtet werden. Man kann aber davon ausgehen, daß sich jüdische Partisanen, die in den sowjetischen Partisaneneinheiten kämpften, nicht anders verhielten als ihre nichtjüdischen Kameraden. Daß die jüdischen Partisanen der Familiengruppen mehr requirierten, als die reinen Kampfeinheiten, liegt auf der Hand. Die 60 Partisanen der Bielski-Gruppe mußten für sich selbst, vor allem aber für die übrigen Angehörigen der Gruppe Beschlagnahmungen vornehmen. Daß sie dabei nicht besonders feinfühlig vorgingen, geht schon aus eigenen Berichten hervor. „It was a messy job [Versorgungsoperationen] that required a willingness to be brutal, a willingness to threaten the life of a peasant who resisted giving up food." 30 Tuvia Bielski bezeichnete nach dem Krieg die „Versorgungsoperationen“ seiner Partisanen offen als „Raubzug.“"31

Die unten abgedruckten Dokumente und Befehle deuten darauf hin, daß auch willkürliche Plünderungen und andere Gewalttaten jüdischer Partisanen nicht selten vorkamen, ähnlich derer der übrigen sowjetischen Partisanen. Siemon Zorin begab sich oft mit einer Gruppe von mindestens 30 berittenen Partisanen in ein Dorf, in dem er sich zuvor ein Mädchen ausgesucht hatte. Er kam in dem Dorf mit seinen Leuten an und erklärte dem Vater des Mädchens, er wolle seine Tochter heiraten und der Vater habe eine „Hochzeit“ für die Partisanen zu organisieren. Widerspruch kam nicht in Frage. Nach zwei drei Tagen verließ Zorin mit seinen Partisanen das Dorf und kehrte zu seiner Abteilung zurück. ${ }^{32}$ Anatol Wertheim, der oft bei diesen „Hochzeiten“ dabei war, plagten nach dem Krieg offenkundig keine Schuldgefühle.

Ein nicht zu unterschätzender Bewegrund für die Übergriffe jüdischer Partisanen war Rache. Unter den sowjetischen Partisanen waren wohl die Juden am meisten traumatisiert und brutalisiert durch die stattfindende systematische Ermordung der jüdischen Bevölkerung. Alle Juden wurden zum Tode verurteilt und zugleich für vogelfrei erklärt. Praktisch jeder Nichtjude konnte sich an ihnen vergreifen. Nicht wenige taten dies. Sie sind dazu angehalten, ja sogar verpflichtet worden. Es ist deshalb nicht weiter verwunderlich, daß sich diejenigen Juden, die zwar überlebt, aber ihre Nächsten verloren hatten, oft nach Rache sehnten, Rache an echten bzw. ver-

29 Gemeindeverwaltung in Niedzwiedzica an den Kommandeur der Sicherheitspolizei und des SD, Hauptaussenstelle Baranoviče (ohne Datum, Ende August 1942), Abschrift/Übersetzung: AAN, F 76 (ohne Paginierung).

30 Duffy, Bielski Brothers, S. 134; vgl dazu auch Tec, Bewaffneter Widerstand, S. 123-125, 173, $180,227,271$.

31 Tec, Bewaffneter Widerstand, S. 151.

32 Wertheim, Żydowska Partyzantka, S. 149. 
meintlichen Tätern, Mittäter oder Nutznießer des Massenmordes an den Juden. ${ }^{33}$ Yakov Segalchick, Partisan der Abteilung „Narodnyj Mstitel'“ (zu deutsch „Volksrächer"), schrieb nach dem Krieg: "The need to get revenge on all the killers without uniforms who were running free, people who were our neighbors in yesterday than who later became our killers, could not let got of me. So I used every free day I had to get revenge. “34

${ }^{33}$ Vgl. dazu Tec, Bewaffneter Widerstand, Duffy, Bielski Brothers.

34 Eternal Testament: Memoirs of a Partisan by Yakov Segalchick. Translated and transliterated by Eilot Gordon. www.shtetlinks.jewishgen.org/Myadel/eternal_testament.htm. 


\title{
Dokumente
}

\author{
Dokument Nr. 1
}

Befehl Nr. 0048 für die Stalin-Partisanenbrigade über die Bekämpfung des
jüdischen Chauvinismus und des Antisemitismus vom 3. Juni 1943

\author{
Vereinigtes Rajonzentrum Ivenec \\ BEFEHL \\ für die STALIN-Partisanenbrigade
}

Streng gebeim

Expl. Nr. 2

3. Juni 1943

Nr. 0048

Pušča N.

Am 12. Mai 1943 ließ die Führung der Dzeržinskij-Partisanenabteilung den ehemaligen Partisanen Grigorij Rivin, Nationalität: Jude, wegen der systematischen Förderung des jüdischen Chauvinismus erschießen, der sich in der Überbetonung dessen ausdrückte, daß man die Juden in der Abteilung nicht möge, gegen sie hetze, sie schikaniere usw.; Rivin war deshalb wiederholt verwarnt worden.

Rivin erfüllte nicht die Forderung der Abteilungsführung, seine überschüssigen persönlichen Waffen - zwei Revolver und eine Mauserpistole - an die Abteilung abzuliefern. Er verletzte die Würde der Kommandeure und Leiter der Partisanenabteilungen, indem er sie unbedarft und Selbstversorger nannte, sprach sich sogar dagegen aus, daß für die Abteilungsführung bessere Speisen zubereitet werden, und drohte den Kommandeuren, mit ihnen nach dem Einzug „der Unsrigen“, d.h. der Roten Armee, abzurechnen.

Rivin handelte als Agitator des jüdischen Chauvinismus, wodurch er in den Reihen der Partisanen Zwistigkeiten verursachte und Unstimmigkeiten in die Beziehungen zwischen den jüdischen und den nichtjüdischen Partisanen hineintrug. Ausgehend vom Dargelegten,

BEFEHLE ICH:

1. Die Erschießung des ehemaligen Partisanen der Dzeržinskij-Abteilung Grigorij Rivin als begründet zu qualifizieren.

2. Die Führung der Dzeržinskij-Abteilung darauf hinzuweisen, daß sie in der Angelegenheit der Erschießung Rivins überstürzt gehandelt hat. Rivin wurde nicht in genügendem Maße des Chauvinismus überführt und von der Allgemeinheit der Partisanen bestraft; außerdem wurde meine Anweisung über das Verbot von Erschießungen ohne entsprechende Erlaubnis des Brigadekommandos übergangen.

3. Ich mache die Kommandeure und Politruk der Abteilungen, die Partei- und Komsomolorganisationen besonders darauf aufmerksam, daß das Einschleppen von 
jüdischem Chauvinismus und in gleichem Maße von Antisemitismus als eine faschistische Methode der Zersetzung der Partisanen einzuschätzen ist; deswegen muß auf jede Äußerung des Antisemitismus oder des jüdischen Chauvinismus entsprechend reagiert werden.

4. Die Führung der Dzeržinskij-Abteilung hat die Tatsache zu untersuchen, daß die Partisanin Gal'a Ragovina meinen Namen als Bevollmächtigter des Vereinigten Rajonzentrums für eigene $Z$ wecke ausnutzte, und mir über die getroffenen Maßnahmen zu berichten.

5. Den Partisanen und Partisaninnen jüdischer Nationalität muß erläutert werden, daß ihre Ansicht über eine angebliche Hetze gegen die Juden auf Einbildung beruht. Es ist ihnen zur Kenntnis zu bringen, daß 10 Partisanen und Partisaninnen jüdischer Nationalität für ihre Tapferkeit, Kühnheit und ihren Heldenmut für Regierungsauszeichnungen vorgeschlagen sind.

6. Es ist auf die Tatsache hinzuweisen, daß der Partisan der Dzeržinskij-Abteilung Iosif Šimanovič, der als Einwohner von Naliboki der Abteilung während des Marsches auf diese Ortschaft den Weg zu zeigen hatte, sich verlief und eine Gruppe von Partisanen nicht rechtzeitig zum Ausgangspunkt führte. Diese Handlung von Šimanovič ist dem Verrat gleichzusetzen, und er hätte deswegen an Ort und Stelle erschossen werden sollen. Aber man ließ ihn am Leben.

Dieser Befehl ist der gesamten Mannschaft der Brigade vorzulesen.

Kommandeur der STALIN-Brigade Leutnant

Kommissar der STALIN-Brigade Oberpolitruk

Stabschef der STALIN-Brigade Oberleutnant
(Gulevič)

(Murašev)

(Karpov)

F. 1329, op. 1, d. 35, 11. 8-9.

\section{Dokument Nr. 2}

Befehl Nr. 023 des Bevollmächtigten des Zentralstabes der Partisanenbewegung und des ZK der KP(b)B für die Oblast' Baranoviči vom 12. Juni 1943 anläßlich des bewaffneten Zusammenstoßes zwischen einem Sprengtrupp der Žukov-Brigade und den jüdischen Partisanen aus der Abteilung „Für Sowjet-Belorußland“

\section{Befebl}

Streng gebeim

des Bevollmächtigten des Zentralstabes der Partisanenbewegung beim Hauptquartier des Oberkommandos und beim ZK der KP(b) Belorußlands für die Oblast' Baranoviči

\section{Nr. 023}

12. Juni 1943

Die durchgeführte Überprüfung stellte fest: Als der von P. V. Kudrjašov befehligte Sprengtrupp der Abteilung Nr. 270 der Žukov-Brigade (die Sprengmeister Guček, Rogošin, Smuškevič, Ščukov und Kurilov) unterwegs war, um einen Auftrag auszuführen, wurde er beim Dorf Mironka irrtümlicherweise vom Wachposten Berkovič aus der Abteilung „Für Sowjet-Belorußland“ der Čkalov-Brigade be- 
schossen. Hierbei wurde der Sprengmeister Gen. Kurilov getötet. Ohne den Vorfall geklärt zu haben, begann der Trupp unter Duldung des Kommandeurs Gen. Šarkevič eine wilde Verfolgung der jüdischen Gruppe.

Kudrjašov tötete alle sieben Mann, und die jüdischen Lager wurden verbrannt. An der barbarischen Abrechnung und der Entwaffnung der jüdischen Partisanen beteiligte sich der Kommandeur einer Gruppe Gen. Boženko, der sich als „Bevollmächtigter des ZK und des Stabes von Platon" ausgab. Eine derart massive Provokation, die ihren Ausdruck in der Vernichtung einer Gruppe jüdischer Partisanen fand, wurde durch den in der Abteilung von Sarkevič und Boženko verbreiteten Antisemitismus ermöglicht, den die Führungen der Abteilungen sowie die Parteiund Komsomolorganisationen nicht bekämpfen. Hiervon ausgehend, befehle ich:

1. Der Kommandeur der Gruppe Gen. Boženko ist wegen der Duldung der Willkürakte bei der Entwaffnung der jüdischen Partisanen, der Verbrennung der jüdischen Lager, des provokatorischen Verhaltens, indem er sich als „Bevollmächtigter des ZK und des Stabes von Platon" ausgab, vom Posten des Kommandeurs abzusetzen, zu verhaften und durch das Vereinigte Rajonzentrum Ivenec gerichtlich zu belangen.

Die Gruppe Boženko ist in die Abteilung von Gen. Šemetovec einzugliedern, die Gruppe von Deserteuren aus der Stalin-Brigade (Levkov, Šajdarov und Volčkov) zu entwaffnen und in die Verfügungsgewalt der Stalin-Brigade zu übergeben, damit entsprechende Maßnahmen getroffen werden.

2. Dem Kommandeur der Abteilung Nr. 270 Gen. Šarkevič ist wegen Unterlassung von Maßnahmen zur Unterbindung der willkürlichen Handlungen eine strenge Rüge zu erteilen; er ist darauf hinzuweisen, daß im Falle der Zulassung solcher und anderer Vorkommnisse, die den Namen des sowjetischen Partisanen schänden, noch strengere Disziplinarmaßnahmen erfolgen werden.

3. Wegen unerlaubter Erschießung der Juden sind der Kommandeur des Sprengtrupps Kudrjašov und sein Trupp durch das Brigadekommando nach dem Standrecht zu verurteilen. Kudrjašov kann sein Leben erhalten, wenn er bis zum 15. Juli mindestens 5 Züge des Feindes zum Entgleisen bringt.

4. Die Abteilungsführung, die Partisanen und Partisaninnen müssen ihre Einstellung zu den Juden verändern und diese bei der Kampftätigkeit richtig einsetzen. Erscheinungen des Antisemitismus sind nicht zuzulassen und auf jede Weise zu unterbinden. Die politische Erziehungsarbeit mit den Mannschaften ist zu verstärken.

5. Der Befehl ist bis Zugführerebene bekannt zu geben.

Bevollmächtigter des Zentralstabes

der Partisanenbewegung und des ZK der KP(b)B

für die Oblast' Baranoviči

(PLATON)

F. 1329, op. 1, d. 29, 1. 20. 


\section{Dokument Nr. 3}

\section{Meldung der Führung der Abteilung Zorin vom 27. August 1943 über den Konflikt mit der Leitung der Pervomajskaja-Brigade (Juli-August 1943)}

Familienabteilung Zorin 27.VIII.1943

\section{Nr. 63}

An den Bevollmächtigten des Belorussischen

Stabes der Partisanenbewegung für das

Vereinigte Rajonzentrum Ivenec

Gen. Dubov

\section{Meldung}

In der zweiten Julihälfte d. J. beschloß die Abteilungsführung, wegen der Verfolgung durch den Gegner für eine Zeitlang aus dem Lager zu einem von der Leitung genannten Ort auszuweichen. Unsere ganze Abteilung zog sich in vollständiger Ordnung zurück. Zugleich zogen sich die Abteilungen der Pervomajskaja-Brigade unter willkürlicher Zurücklassung des Materials der Abteilungen zurück. Drei Tage nach dem Rückzug kehrte eine erste Gruppe von Partisanen der Abteilung auf Befehl des Abteilungskommandeurs ins Lager zurück mit dem Auftrag, die Truppenwirtschaft wieder vollständig in Ordnung zu bringen. Neben den eigenen Lebensmitteln und dem Vieh (2 Kühe) brachte die Gruppe 18 Kühe und 35 Pud Getreide aus der Abteilung Bel'skij in Abstimmung mit der Führung dieser Abteilung mit. Nur die Abteilungspferde (18 St.) konnten nicht vollzählig aufgefunden werden, und die Gruppe trieb 20 von den vielen Pferden, die sich auf den Straßen und im Moor befanden, ins Lager.

Zugleich kehrten Gruppen von Partisanen der Pervomajskaja-Brigade allmählich in ihre Lager zurück. Anstatt die Versorgungstrupps wiederherzustellen, die Sachen der Abteilung zu sammeln und das Vieh, das sich in großer Zahl im Moor befand, in die eigenen Lager zu treiben, kamen einzelne Partisanen der Brigade in unsere $\mathrm{Ab}$ teilungen und veranstalteten unter dem Vorwand „Die verfluchten Juden haben uns ausgeraubt" Aufruhr, stießen Drohungen aus und verlangten unter Androhung von Gewalt die Rückgabe der geraubten Sachen. Es kam so weit, daß 2 Partisanen der Pervomajskaja-Brigade in unserem Lager ohne Umschweife erklärten, die Partisanen müßten die Sache, die die Deutschen mit den Juden begonnen haben, zu Ende führen. Das Brigadekommando traf keine Maßnahmen dagegen. Mehr noch, der Stabschef der Brigade persönlich erlaubte es sich, in unser Lager zu kommen und die Rückgabe von 5 Kühen zu verlangen, die in der Abteilung Markov „geraubt“ worden seien. In seiner Anwesenheit nannten die Partisaninnen der Abteilung Markov die Kämpfer unserer Abteilung „räudige Judenhunde“, Plünderer, Diebe und gebrauchten andere ähnliche Schimpfwörter.

Als der Abteilungskommandeur Zorin die Auslieferung seiner Kühe verweigerte, drohte der Stabschef der Brigade dem Abteilungskommandeur, daß er ihn wie einen Hund fesseln, daß es Opfer geben werde, und schickte sogar einen schriftlichen Befehl an den Brigadestab ab, sofort einen Zug mit einem Maschinengewehr herkommen zu lassen, damit [...]. Um das zu vermeiden, mußten sie 5 Kühe der Abteilung Markov abgeben. In einer Unterredung mit dem Stabschef unserer Abteilung erklärte der Stabschef der Brigade, er verstehe ausgezeichnet die antisowjetische $\mathrm{Be}-$ wegung unter den Partisanen, verstehe dagegen nicht, wozu Zorin seinen „Kram“ 
alten Leuten, Frauen und Kindern wegnehme, wo doch so viele Helden an der Front den Tod fänden. Als der Stabschef unserer Abteilung in dienstlichen Angelegenheiten in den Stab der Pervomajskaja-Brigade kam und dort vor dem Stab sein Reitpferd stehen ließ, nahm ein Partisan der Pervomajskaja-Brigade den Sattel als "geraubt" an sich, obwohl der Sattel von einem Sattler unserer Abteilung angefertigt war. Der Stabschef der Brigade tat nichts, um den Sattel zurückgeben zu lassen. In den ersten Augusttagen hielten mehrere Partisanen der Brigade unseren Nachrichtensoldaten, der einen Auftrag hatte, fest, verprügelten ihn und nahmen ihm den Sattel und das Pferd weg. Zugleich hat der Leiter der Stabsaufklärung das Reitpferd unserer Streife [...]. Das Verhalten des Brigadekommandos hat die Partisanen der Brigade so sehr beeinflußt, daß bei jeder Begegnung mit den Kämpfern unserer Abteilung die Worte „schmutziger Jude“, Dieb, Plünderer usw. fielen. Es kam so weit, daß jederzeit ein [...] zu erwarten war, und nur weil unsere Abteilung in ein anderes Lager zog, haben wir das vermeiden können.

Jetzt, wo die ganze Abteilung gemäß Befehl des Rajonzentrums bei Feldarbeiten eingesetzt ist, wo die Partisanen der Abteilung, die 11 Stunden am Tag arbeiten, nur Kartoffeln essen, weil sie kein einziges Gramm Mehl haben und die 19 Kühe als ihren „eisernen Vorrat" betrachten, kommt ein ultimativer Brief des Kommandeurs der Pervomajskaja-Brigade, das „Geraubte“ zurückzugeben. Das Ziel des Briefes ist klar: uns die letzten Kühe und Pferde wegzunehmen und das Leben der Abteilung lahmzulegen.

In Anbetracht des Gesagten ersuche ich Sie, Gen. Dubov, zusammen mit Gen. Platonov der Pervomajskaja-Brigade zu befehlen, daß sie ihre ungesetzlichen Handlungen gegenüber unserer Abteilung einstellt.

Abteilungskommandeur

Abteilungskommissar

Stabschef
(Zorin)

(Fejgelman)

(Vertgajm)

F. 1329, op. 1, d. 32, 11. 38-39.

\section{Dokument Nr. 4}

\section{Bericht über Vorkommnisse im Dorf Dubniki \\ (17.-18. November 1943) (Auszug)}

An den Bevollmächtigten des ZK der KP(b)B und des Belorussischen Stabes der Partisanenbewegung Gen. Dubov

\section{Bericht}

Habe zu melden, daß die in Ihrem Auftrag durchgeführte Untersuchung im Fall des Mordes an 11 Personen aus der Gruppe Zorin im Dorf Dubniki (Rajon Ivenec) folgendes ergeben hat: Das Verbrechen wurde in Wirklichkeit von polnischen Legionären begangen, und daran beteiligten sich in Wirklichkeit Leutnant Norkevič 
(alias Noč) $)^{35}$ und sein Stellvertreter Jakubovič (alias Dub). Im Dorf Ročeviči besuchten wir den Bürger Voropaj, und aus den Äußerungen von dessen Sohn ging hervor, daß jener vom Mord gewußt hatte. Er erklärte, daß der Befehl zur Ermordung der Festgenommenen von Zorin nur über Leutnant Noč und seinen Stellvertreter Dub übermittelt wurde. Während wir uns in Voropajs Haus befanden, traf eine Gruppe von Legionären ein, darunter der ehemalige Vorsitzende des Mnišnskij-Dorfsowjets Filipovič. Als ich ihm sagte, daß die Juden plündern und deswegen bestraft werden sollten, antwortete er mir: „Wir haben sie schon bestraft.“ Als wir im Dorf Dubniki, am Ort des Mordes, ankamen, erklärte der Bürger Valerian Širvel', in jener Nacht seien tatsächlich Legionäre im Dorf gewesen. Im benachbarten Dorf Sobkovščina befand sich eine 12 Mann starke Gruppe von Zorin, denen die Polen 6 Pferde mit Fuhren wegnahmen; die Gruppe ließen sie gehen. Die Pferde, die den Zorin-Leuten gehört hatten, wurden ins Dorf Dubniki befördert. Nach einiger Zeit stellten sich die Zorin-Leute ein und verlangten ihre Pferde von den Polen zurück, wurden jedoch von einem polnischen Posten festgehalten. Darauf kam eine starke polnische Gruppe, sie kreiste die Partisanen von Zorin ein und entwaffnete sie. Der stellvertretende Kommandeur der Schwadron Jakubovskij befahl, alle zu erschießen, doch nach einiger Zeit wurde beschlossen, sie zuerst aus dem Dorf weggehen zu lassen, um sie dann einzuholen und zu erschießen, was am 20 . November 1943 im nächsten Wald auch geschah, Leichen aber wurden nicht gefunden. [...]

F. 3601, op. 1, d. 8, 1. 79.

\section{Dokument Nr. 5}

Protokoll des vom Partisanen M. Vaker am 24. November 1943 angestellten Verhörs in Sachen der Ermordung von 10 Partisanen der Abteilung Zorin im Dorf Dubniki

\section{Verhörprotokoll}

Ich, Partisan Mečislav Vaker, habe am 24.11. 43 in Anwesenheit des Partisanen Michail Petrov und des Stabschefs Anatolij Verdgajm [Anatol Wertheim, B.M.] aus der Abteilung Zorin den Partisanen Černjak aus der Abteilung Zorin über den Fall der Ermordung von 10 Partisanen der Abteilung Zorin im Dorf Dubniki, IvenecRajon, verhört.

Černjak, Lev Grigor'evič, geb. 1919 in Minsk, soziale Herkunft: Arbeiter, sozialer Stand: Partisan der Abteilung Zorin, Posten: Kämpfer, Gemeiner, Beruf: Elektromonteur. In der Abteilung ab 27.6. 43. In die Abteilung aus dem Ghetto der Stadt Minsk gekommen.

Frage: Wann habt ihr das Lager verlassen und in welcher Richtung?

35 Gemeint ist Zdzisław Nurkiewicz, der jedoch in einem Gerichtsverfahren in Polen in den $60 \mathrm{er}$ Jahren von diesem konkreten Vorwurf freigesprochen wurde. Näheres bei Zygmunt Boradyn, Niemen rzeka niezgody. Polskosowiecka wojna partyzancka na Nowogródczyźnie 1943-1944, Warszawa 1999, S. 155. 
Antwort: Wir verließen das Lager am 16.6. 43 zur einer wirtschaftlichen Operation und kamen im Dorf Dubniki am 17.6. 43 nachts an.

Frage: Was tatet ihr in jener Nacht im Dorf Dubniki?

Antwort: Im Dorf Dubniki warnten uns die Legionäre, daß sich dort ihre Abteilung befand, wir sollten ins nächste Dorf umziehen, und so begaben wir uns ins Dorf Sobkovščina, ein Kilometer weit von Dubniki entfernt, und gingen an die Durchführung der Operation. Nach kaum 40 Minuten wurde das Dorf Sobkovščina aus Maschinengewehren und Degtjarev-Maschinenpistolen aus der Richtung Dubniki beschossen. Wir mußten die Fuhren stehen lassen und fliehen, weil das Feuer direkt auf uns gerichtet war, aber Tote und Verwundete gab es unter uns nicht. Unsere Gruppe bestand aus 14 Mann. Einer von den Unsrigen verlief sich während des Beschusses und kehrte ins Lager zurück.

Frage: Wie habt ihr erfahren, daß ihr tatsächlich von Legionären beschossen wur$\operatorname{det}$ ?

Antwort: Wir flohen aus dem Dorf weniger als einen Kilometer weit. Nach einiger Zeit schickten wir einen Spähtrupp ins Dorf Sobkovščina. Er stellte fest, daß im Dorf Legionäre waren und den Bauern erklärten: Wenn das Partisanen waren, sollen sie nach Dubniki zurückkommen, wir werden ihnen die Pferde zurückgeben. Und so gingen wir dorthin. Kurz vor dem Dorf wurden wir von Wachen aufgehalten, und die schrien: „Das ist doch die Bande, die wir auseinandergetrieben haben." Die Legionäre forderten einen Partisanen unserer Gruppe, Volkov, auf, zu ihnen zu Verhandlungen zu kommen, aber von diesen Verhandlungen kam er nicht zurück.

Frage: Was habt ihr zu tun beschlossen, als euer Partisan nicht zurückkam?

Antwort: Dann riefen wir nach dem Kommandeur der polnischen Abteilung (den Familiennamen kenne ich nicht, er wurde Chef genannt). Der Kommandeur der Polen kam nicht, statt seiner erschienen mehr als 40 Reiter. Sie kreisten uns ein und forderten von uns, die Waffen abzuliefern. Wir entwaffneten uns jedoch nicht, und sie schlugen auf uns mit Peitschen und Zügeln ein. Dabei wurde dem Partisanen Krasnic mit dem Lauf einer Maschinenpistole der Schädel eingeschlagen und dem Partisanen Sagal'čik der rechte Kiefer gebrochen. Sie wurden vom Chef (dem „Dicken") persönlich verprügelt.

Frage: Was geschah, nachdem ihr verprügelt worden wart?

Antwort: Während der Prügelei floh ein Partisan unserer Gruppe, Šivanovič, ließ dabei seinen Zehnlader und seinen Revolver bei der polnischen Abteilung zurück und flüchtete sich ins Lager. Nach der Prügelei wurden wir alle durchsucht, sie nahmen uns jedoch nur die Waffen weg, ließen uns paarweise antreten und ins Haus des Bürgers Sirnel gehen.

Frage: Was geschah in diesem Haus, nachdem ihr dorthin abgeführt worden wart?

Antwort: Sobald wir im erwähnten Haus waren, wurde da ein Posten aufgestellt. Nach einer Stunde kamen die Legionäre, befahlen uns, unsere Stiefel auszuziehen, verprügelten uns wieder und zogen uns die Stiefel aus, stattdessen gaben sie uns ihre zerrissenen Stiefel. Nach einer weiteren Stunde erschien ein Legionär, der Pan Zev hieß. Als er eintrat, standen wir nicht auf [...], deshalb verprügelte er uns. Er teilte uns in zwei Reihen, die Kommandeure und die Vostočniki jeweils für sich. Er fragte uns [...], weshalb wir Krieg führen. Wir antworteten, daß wir für die Sowjetmacht kämpfen. Bei dieser Antwort schlug er erneut auf uns ein und sagte dabei, daß sie 
für ein Polen ohne Juden und Kommunisten kämpften. Dann fragte er die Vostočniki nach Familien- und Vornamen, Geburtsjahr und Parteizugehörigkeit. Den Komsomolzen [...] verprügelte er, die übrigen gaben nichts zu, darauf begann er aus [...] zu [...]. Darauf fragte er, wer uns verprügelt hatte, und befahl der Hausbesitzerin, uns Tücher zu geben, damit wir uns abtrockneten, und befahl, uns nicht mehr zu schlagen, [...] versetzte dem Legionär mehrere Schläge und ging. Auf Befehl des Kommandeurs gaben uns die Bauern zu essen, danach betrat ein Legionär in einer Lederjacke das Haus, man nannte ihn Pan Oberleutnant. Er befahl uns, unsere Sachen abzulegen, was wir auch taten. Weil sich ihm unser Partisan Sagal'čik nicht schnell genug auszog, schlug der erwähnte Oberleutnant mit dem Gewehr auf ihn ein und brach ihm den Arm. Darauf wurden wir auf Gold und Uhren durchsucht, doch solches hatten wir nicht. Ich persönlich hatte meine Uhr im Bett des Bürgers Mil'vel' versteckt. Nach der Verprügelung wies man uns an, unsere Sachen wieder anzulegen, und das taten wir. Danach ging einer der Legionäre aus diesem Raum in die Küche, sprach mit einem anderen, kehrte mit einem Strick zurück und gab ihn dem Oberleutnant in der Lederjacke. Der Oberleutnant band zwei Kämpfer mit diesem Strick an Händen, die übrigen wurden von den Legionären gefesselt, und sie führten uns aus dem Hause nach draußen.

Frage: Wohin führten sie euch und was sagten sie, warum veränderte das alles?

Antwort: Sie sagten uns, daß die Juden die letzten Kühe wegnehmen, „Wir haben euch zwei Jahre lang mit Lebensmitteln versorgt, jetzt aber ist deshalb Schluß damit, und nun fahren wir euch ins Rajonzentrum", aber unsere Fuhren und Waffen beließen sie da. Als wir durchs Dorf geführt wurden, sagten die Bauern unter Hohngelächter, nun sei Schluß mit der Kommune. Unterwegs wurden wir eigens gezwungen, durch den Sumpf zu gehen; uns begleiteten 5 Legionäre und der Oberleutnant in der Lederjacke. Als wir höchstens einen halben Kilometer vom Dorf entfernt waren, bogen sie nach links ab, in den Wald. Gegen 12 Uhr waren wir an dem Ort angelangt, wo die Erschießung stattfand. Sie sagten uns, wir sollten uns mit dem Rücken zu ihnen stellen, und als wir uns umdrehten, schossen die Legionäre. Den ersten Schuß, mit dem der Partisan Šelkov getötet wurde, gab der Oberleutnant in der Lederjacke ab. Als Šelkov hinfiel, ließ ich mich auch fallen. Nach mir fielen auch meine Kameraden hin. Darauf kamen die, um zu prüfen, ob auch alle tot waren. Einer kam auf mich zu, stieß meinen Kopf mit dem Fuß, sagte, der lebt noch, und schoß auf meinen Kopf, aber er durchschoß nur die Mütze, ohne mich zu treffen. Danach stießen sie noch ein paar Schimpfwörter aus und gingen. Nach kaum einer halben Stunde stellten sich Bauern ein und wollten uns die Stiefel ausziehen, da kam ich zu mir und hob den Kopf. Die Bauern rannten weg, da stand ich auf, band mir den Arm los und flüchtete auch. Ich erreichte das Gehöft Slobodka, im Haus des Bürgers Anton Gudtok zog ich mich um, wusch mich und ging in Richtung Gryn'. Unterwegs begegnete ich dem Stabschef der Frunze-Brigade Lojko. Ich erzählte ihm all das und begab mich nach Gryn'. Dort fand ich unsere Partisanen vor und fuhr mit ihnen ins Lager. An Waffen hatten wir nur ein Gewehr, einen Zehnlader, 2 Revolver, eine Pistole, ungefähr 6 Handgranaten und rd. 500-600 Patronen.

Das Protokoll ist mir verlesen worden, was ich durch meine Unterschrift bestätige.

F. 3601, op. 1, d. 8, 11. 70-71. 


\title{
Dokument Nr. 6
}

\section{Bericht über die Kampftätigkeit der Ordžonikidze-Abteilung}

(Ende Herbst 1943?)

\author{
Bericht \\ über die Kampftätigkeit der Partisanenabteilung \\ „S. Ordžonikidze“
}

Die jüdische Abteilung entstand im Juli 1941, als die Deutschen kamen. Anatolij Davidovič Bel'skij, der sich vor ihren Verfolgungen als ehemaliger Bevollmächtigter des Volkskommissariats für Eisenindustrie für die Oblast' Vilejka und einen Teil der Oblast' Baranoviči vor ihnen versteckt gehalten hatte, kehrte in sein Heimatdorf Stankeviči zurück und ging daran, eine Partisanenabteilung zu organisieren. Er wußte, daß die deutschen Okkupanten in erster Linie barbarisch gegen die jüdische Nationalität vorgingen, und so setzte sich Anatolij Davidovič das Ziel, eine jüdische Partisanenabteilung aufzustellen, um aktiv gegen den blutrünstigen Faschismus zu kämpfen und zugleich die Menschen vor der unvermeidlichen Ermordung durch die deutschen Okkupanten zu retten. In erster Linie zog er seine drei Brüder, seine Verwandten und Bekannten, insgesamt 20 Mann, zur gemeinsamen Tätigkeit heran.

In der ersten Periode ihrer Tätigkeit tat die Gruppe Folgendes:

1. Politische Agitationsarbeit in der örtlichen Bevölkerung,

2. Beschaffung von Waffen für alle Abteilungsmitglieder,

3. Vernichtung einzelner Volksfeinde (Polizisten, deutsche Spione usw.) im Operationsraum der Abteilung,

4. Vernichtung staatlicher Landgüter.

Im Sommer 1942, als die Partisanenbewegung auf dem Territorium Westbelorußlands zu wachsen begann, stellte Gen. Bel'skij, um die zahlenmäßige Stärke und somit auch die Kampffähigkeit seiner Abteilung zu erhöhen, Kontakte mit den Ghettos von Novogrudok, Lida und anderen her, warb junge, kampffähige Leute an, bewaffnete sie allmählich und entwickelte eine Partisanentätigkeit in breiteren Ausmaßen. Im September 1942 nahm A. D. Bel'skij Verbindung zum Leutnant S. I. Pančenkov auf, der mit seiner Abteilung im einem benachbarten Rajon operierte, und führte zusammen mit ihm mehrere Kampfoperationen durch.

Am 16. 10. 1942 wurde auf der Chaussee Novogrudok - Novoel'naja ein Hinterhalt gegen die deutschen Gendarmen angelegt. Ergebnis: mehrere Deutsche getötet, ein Wagen vernichtet, 2 Maschinengewehre, 3 Gewehre und mehrere Tausend $\mathrm{Pa}$ tronen erbeutet, wobei keine eigenen Verluste zu verzeichnen waren. Am 26. 10. 1942 erfolgte ein Angriff auf die Eisenbahnausweichstelle Jacuki, wo ebenfalls mehrere Deutsche getötet wurden. Am 8. 12. 1942 ein Hinterhalt an der Strecke Jacuki Novoel'naja angelegt, 2 Deutsche getötet, zwei Gewehre mit Patronen erbeutet. Am 12. März 1943 Teilnahme am Gefecht gegen die Polizei im Dorf Dračilowo: 15 Polizisten getötet bzw. verwundet. Am 19. März 1943 wurde die Fernsprechverbindung an der Chaussee Novogrudok - Lida und Novogrudok - Vseljub in einer Länge von $5 \mathrm{~km}$ heruntergerissen. Außerdem wurde der Rajon in dieser Periode von deutschen Spionen und Provokateuren gesäubert, staatliche Landgüter, in denen Polizeireviere hätten eingerichtet werden sollen, vernichtet, usw. In der Zeit der Tätigkeit der Abteilung wurden 14 Hitlerleute, 17 Polizisten, 33 deutsche Spione 
vernichtet, gemeinsam mit der Abteilung von Gen. Groznyj eine Eisenbahnbrücke in die Luft gesprengt, Telegrafenmaste auf einer Strecke von 6 Kilometern gefällt, 8 staatliche Landgüter sowie ein großes Sägewerk niedergebrannt, u.a. Die Abteilung erfüllte alle jüngsten Aufträge der Regierung und nahm in der Brigade den ersten Platz ein. An der Strecke Baranoviči - Lida wurden rd. 700 Meter Gleise verbrannt. Am 10.6. 1943, als sich die Abteilung in den Jasenevskie-Wäldern aufhielt, wurde eine Razzia veranstaltet. Im Lager befanden sich damals etwa 700 Personen. Dank der exakten Arbeit der Aufklärung und der getarnten Posten gelang es, alle ohne Verluste an einen sicheren Ort zu bringen, mit Ausnahme von 2 Wachen, die den Feind bemerkt und Feuer auf ihn eröffnet hatten, so daß sie den Deutschen, die sie umzingelten, nicht mehr entgehen konnten. Nach diesem Überfall begab sich die Abteilung in die Naliboki-Pušča, um dort wieder ein Lager für die Familien der Abteilungsmitglieder einzurichten, während die Kampfgruppe der Abteilung in ihren Operationsraum hätte zurückkehren sollen. In dieser Zeit wurde in der NalibokiPušča eine große Razzia veranstaltet. Die Abteilungsführung mußte die Abteilung in Gruppen aufteilen und sie aus der Pušča herausführen. Hierbei hatte die Abteilung keine Verluste, nur ein Partisan ertrank während des Übersetzens über den Fluß Bereza. Nach der Razzia wurde die Kampfeinheit in ihrem Operationsraum versammelt. Die Abteilung operierte unter der Leitung der Kirov-Brigade und mußte umorganisiert werden: Die Kampfeinheit sollte für sich bestehen, die Familiengruppe aber in der Pušča bleiben. In dieser Zeit trafen Juden ein, die aus dem Ghetto von Lida geflohen waren, und stießen zur Abteilung. Hinzu kamen noch alle Juden, die aus dem Ghetto von Novogrudok geflohen waren, etwa 200 Personen. Der Abteilungskommandeur A. D. Bel'skij sammelte alle Angehörigen, die unbewaffnet waren, und führte sie in die Pušča. Hier ging man sofort daran, eine Basis für diese Leute einzurichten. Es wurden Kartoffeln geerntet, eine Mühle, eine Bäkkerei, eine Metzgerei, mehrere Schustereien, Schneidereien, Sattlereien, Klempnereien u.a. Werkstätten eingerichtet. Das nahm 3 Wochen in Anspruch. Danach kehrte der Abteilungskommandeur A. D. Bel'skij in seine Abteilung zurück. Der Stab der Kirov-Brigade hatte inzwischen den Kommandeur zu sich beordert, und da er den Grund seiner Abwesenheit nicht kannte, wurde zum Abteilungskommandeur der ehemalige Stellvertreter Hauptmann Ljašenko ernannt, der zusammen mit dem Stabschef in die Abteilung entsandt wurde, um dem Kommandeur bei deren Umorganisierung zu helfen. A. D. Bel'skij wurde die Familiengruppe anvertraut, die er in die Naliboki-Pušča gebracht hatte; er wurde zu ihrem Kommandeur ernannt. Maßnahmen wurden getroffen, um die kampffähigen Abteilungsmitglieder zu bewaffnen.

A. D. Bel'skij wurde in die Oblast' Minsk entsandt und konnte dort eine Anzahl von Waffen beschaffen. Gegenwärtig zählt die in der Pušča liegende Abteilung rd. 300 Personen, davon sind etwa 200 bewaffnet. Die Waffen der Abteilung: 2 Degtjarev-Maschinenpistolen, 8 halbautomatische Gewehre, der Rest sind Flinten.

Der politisch-moralische Zustand der Abteilung ist ausgezeichnet. Die Abteilung ist um ihren Kommandeur und ihre Führung geschlossen und erfüllt mit Freude alle übertragenen Aufgaben. Die Partisanen sind über die internationale politische Lage informiert und streben danach, sich an unserem Erzfeind, dem deutschen Faschisten, zu rächen.

In der Abteilung besteht eine junge Komsomolorganisation, die [...] Mitglieder 
zählt. Die Abteilungsführung unterhält ständige enge Kontakte mit der örtlichen Bevölkerung, im Rajon gibt es uns ergebene Leute, die Sonderaufträge der Abteilungsführung erfüllen. Die Abteilung begeht alle großen Revolutionsfeste stets zusammen mit der örtlichen Zivilbevölkerung, veranstaltet dabei Kundgebungen, Aussprachen und Konzerte von Laienkünstlern. In letzter Zeit kam es zu keinem Fall des Marodierens der Partisanen in der Zivilbevölkerung. Die Führung sorgt ständig dafür, daß sich die Partisanen zur Zivilbevölkerung richtig verhalten.

F. 3623, op. 1, d. 2, 11. 17-20.

\title{
Dokument Nr. 7
}

Bericht der Führung der Familiengruppe der Ordžonikidze-Abteilung vom 5. Dezember 1943 über die geleistete Arbeit

\author{
Kirov-Partisanenbrigade \\ Ordžonikidze-Abteilung \\ Familiengruppe \\ Pušča N. \\ 5. 12.43 \\ Nr. 0020
}

Streng geheim

\section{Bericht an den Kommandeur der Kirov-Partisanenbrigade Hauptmann Vasil'ev über die geleistete Arbeit}

Am 17. September 1943 bildete ich eine Familiengruppe (250 Personen), ließ die Kampfeinheit mit dem ganzen Stab im Operationsraum liegen und begab mich in Anbetracht der Tatsache, daß der Aufenthalt der Abteilung mit Familien im Kampfgebiet unmöglich war, und auf Weisung des Brigadestabs mit ihnen zur alten Basis in der Naliboki-Pušča. Bei der Ankunft fand ich dort die von mir zurückgelassene Gruppe von 36 Personen vor, die inzwischen auf 63 Personen angewachsen war. Sie ernteten Kartoffeln und bauten ein Sommerlager. Ich meldete mich beim Bevollmächtigten des $\mathrm{ZK}$ der $\mathrm{KP}(\mathrm{b}) \mathrm{B}$ für das Vereinigte Ivenec-Rajonzentrum, in dessen Gebiet sich das Lager befindet, und ging an die Einrichtung einer Basis für etwa 700 bis 800 Personen. Außerdem erhielt ich Weisung, alle Familienangehörigen, die sich in der Pušča und in den Rajons Stolbcy und Mir befanden, etwa 100 Personen, zu sammeln. Sie begannen sofort mit der geplanten Kartoffelernte und mit der Einrichtung des Winterlagers. In Anbetracht unserer Lage begannen wir sofort mit dem Bau einer Bäckerei und einer Mühle, da die ganze Pušča während der Razzia niedergebrannt worden war. Außerdem nahmen wir den Bau verschiedener Werkstätten in Angriff: einer Schusterei, einer Werkstätte für die Herstellung von Fußlappen, einer Waffenschmiede, einer Tischlerei, einer Sattlerei, einer Metzgerei usw. Sie beschäftigen zurzeit über 100 Personen. Die Bäckerei und die Metzgerei versorgen nicht nur die eigene Abteilung und die in der Nähe liegenden Brigaden, sondern 
auch andere Abteilungen. Die Gruppe hat ein Winterlager eingerichtet, dessen Plan ich beilege. Jetzt ist das Lager schon fertig, und die Gruppe baut nun ein zusätzliches, befestigtes Winterlager. Am 30. Oktober wurde die letzte Gruppe von Familieangehörigen der Partisanen hergeführt, sie zählt gegenwärtig 600 Personen, einige sind außerdem in weiteren 4 Lagern beschäftigt, da überall noch verschiedene Arbeiten im Gange sind. Heute hat die Gruppe folgende Vorräte: rd. 200 Tonnen Kartoffeln, $3 \mathrm{t}$ Kohl, $5 \mathrm{t}$ Rüben, $5 \mathrm{t}$ Getreide und sonstiges. An Fleisch haben wir rd. $3 \mathrm{t}$, an Würsten etwa $1 \mathrm{t}$. Ein Teil davon ist in Verstecken untergebracht. Für den täglichen Verbrauch haben wir noch Mehl. Es gibt eine gemeinsame Küche, die dreimal täglich warme Speisen für die ganze Gruppe zubereitet. Drei Gruppen, insgesamt 60 Personen, führen in verschiedenen Rajons wirtschaftliche Operationen durch. Den täglichen Dienst verrichten 66 mit Gewehren bewaffnete Personen, ein Spähtrupp von 6 Personen und 4 Verbindungsleute. In der Basis gibt es keine Seuchen, keine ansteckenden Krankheiten, im Lazarett befinden sich 16 Personen, es wird von einem Arzt und zwei Krankenschwestern betreut.

Kommissar der Familiengruppe

Gehilfe des Stabschefs

(Bel'skij)

(Mal'bin)

F. 3623, op. 1, d. 2, 11. 94-95.

\section{Dokument Nr. 8}

\section{Schreiben der Leitung der Frunze-Brigade an den Kommandeur der Ordžonikidze-Abteilung mit der Forderung, dem Marodieren seitens jüdischer Partisanen ein Ende zu setzen (25.-27. Dezember 1943)}

An den Kommandeur der Ordžonikidze-Abteilung

Gen. Bel'skij

Anbei die Meldung des Garnisonsdiensthabenden der Frunze-Brigade und eine Akte eines Bürgers der Ortschaft Naliboki. Auch melde ich zum zweiten Male, daß Ihre Partisanen mit dem Partisanen Icka Rubeževskij an der Spitze Banditentum treiben. Der erste Plünderungsfall fand auf dem Gehöft des Bürgers Šemet statt, der zweite betraf den Bürger Korženko in der Ortschaft Naliboki. Im ersten Fall, bei der Ausplünderung von Šemet, wurden zwei Ihrer Partisanen, Icka Rubeževskij und Šimanovič, verhaftet und entwaffnet. Gen. Bel'skij, Sie selbst haben dem Brigadekommando Ihr Ehrenwort gegeben, daß solche Handlungen Ihrer Partisanen nicht mehr vorkommen werden, daß Rubeževskij bestraft werden soll und niemand von der Brigade ihn in diesem Rajon jemals wiedersehen wird. Sie haben Ihr Wort nicht gehalten, und die verbrecherischen Handlungen Ihrer Partisanen mit $\mathrm{Ru}$ beževskij an der Spitze dauern an. Solche Handlungen sind im Weiteren unzulässig und dürfen nicht andauern. Ich weise an, daß der geschlachtete Eber sofort dem Bürger der Ortschaft Naliboki zurückgegeben, jede wie auch immer geartete Lebensmittelbeschaffung im Raum Naliboki verboten und der Bandit Icka Rubeževskij bestraft wird. Sollte er sich in diesem Rajon noch einmal zeigen, so wird er 
an Ort und Stelle erschossen. Die übrige Gruppe wird entwaffnet werden und bekommt ihre Waffen nicht zurück.

Den Vollzug in dieser Angelegenheit spätestens am 28.11 (sic!) 1943 melden.

Kommandeur der Frunze-Brigade

Oberleutnant Ključko

Kommissar der Frunze-Brigade Hauptmann Korobkin

F. 3623, op. 1, d. 2, 1. 69 .

\section{Dokument Nr. 9}

Befehl Nr. 001 des Bevollmächtigten des Zentralstabes der Partisanenbewegung und des ZK der KP(b)B für die Oblast' Baranoviči über die Ausgliederung der Abteilung Bel'skij aus der Kirov-Brigade vom 3. Januar 1944

Expl. Nr. 2

Streng gebeim

3. Januar 1944 Nr. 001

Pušča N.

1. Die Reserveabteilung Bel'skij ist aus der Kirov-Brigade auszugliedern, zu einer selbständigen Abteilung zu formieren und unmittelbar meinem Gehilfen Gen. Sokolov zu unterstellen.

2. Als Abteilungskommandeur bestätige ich Gen. Bel'skij, Anatolij Davidovič, als Stabschef Gen. Mal'bin, Lazar' Abramovič, als zeitweiligen Vertreter des Kommandeurs für politische Arbeit Gen. Gordon, Tjanchel' Efimovič.

3. Die Reserveabteilung hat sich auf die Naliboki-Pušča zu stützen. Die wirtschaftlichen Operationen sind im Rajon Novogrudok durchzuführen. Das genaue Verzeichnis der Dörfer, die zum Tätigkeitsraum der Abteilung gehören, wird von Gen. Sokolov festgelegt.

4. Ich stelle der Abteilungsführung die Aufgabe, den in der Abteilung befindlichen Familienangehörigen Lebensmittel und bewaffnete Bewachung zu sichern. Maßnahmen zur Beschaffung von Waffen und zur Bewaffnung der Kampffähigen zu treffen. Die Unbewaffneten energisch bei der Vernichtung der Nachrichtenverbindungen, Brücken, bei der Beschädigung von Straßen und der Aufklärung einzusetzen. Die 150 Bewaffneten zu mindestens 2 bis 3 Diversionsgruppen zu organisieren und die Gleise sowie Militärzüge, Kraftfahrzeuge und sonstige Technik auf den Chausseen zu sprengen, Hinterhalte anzulegen, um die Mannschaften zu töten und die Technik des Feindes zu zerstören.

Bevollmächtigter des Zentralen Stabes

der Partisanenbewegung und des ZK der KP(b)B

für die Oblast' Baranoviči

Generalmajor

(Platon)

F. 3423, op. 1, d. 1, 1.1. 


\section{Dokument Nr. 10}

\section{Schreiben des Bevollmächtigten des Zentralstabes der Partisanenbewegung und des ZK der KP(b)B für die Oblast' Baranoviči vom 12. Januar 1944 zur Erläuterung der Gründe von A. Bel'skijs Absetzung}

\section{Vasilij Efimovič,}

die Materialien über die unrichtige Absetzung von Bel'skij schicke ich zurück, weil ich für die Kirov-Brigade nicht zuständig bin. Mit dieser Frage hat sich Gapeev zu befassen. Zudem befindet sich das ganze Material, das Bel'skij kompromittiert, in der Kirov-Brigade. Ich meinerseits bin der Meinung, daß es richtig war, Bel'skij abzusetzen. So befaßte sich Bel'skij nicht mit Kampfhandlungen, sondern machte in der Abteilung nur seine Geschäfte, erhielt von seinen Partisanen Gold zum Waffenankauf; er eignete sich das Gold an, lieferte jedoch keine Waffen. Ich würde vorschlagen, Bel'skij das Gold an den Staat abliefern zu lassen, er hat nämlich über ein Kilogramm Münzen zaristischer Prägung; dann sollte dieser Bel'skij verhaftet und vor Gericht gestellt werden.

Mit Gruß

\section{1.44 .}

Stepan.

Resolution:

An Gen. Sokolov

Der Sekretär des Oblastparteikomitees Gen. Černyšev erteilt Ihnen den Auftrag, alle Materialien in Sachen des Abteilungskommandeurs Bel'skij zu untersuchen und die notwendigen Maßnahmen zu treffen. Die Ausführung ist zu melden.

Stellv. Leiter der Organisationsabteilung Instruktion

des Oblastkomitees der $\mathrm{KP}(\mathrm{b}) \mathrm{B}$

18. 1. 44.

F. 1329, op. 1, d. 45, 1. 26.

\section{Dokument Nr. 11}

\section{Beschluß der Führung der Abteilung Bel'skij vom 5. Februar 1944 zum Fall I. Kesler}

\section{Beschlu $\beta$}

Am 5. Februar 1944 haben wir, die Unterzeichneten Bel'skij, Anatolij Davidovič, Kommandeur der Partisanenabteilung Bel'skij; Gordon, Tanchel' Efimovič, i. A. Stellvertreter des Kommandeurs für politische Arbeit; und Mal'bin, Lazar' Abramovič, Stabschef, in Abwesenheit des Bevollmächtigten der Sonderabteilung der Abteilung alle Materialien zum Fall Kesler, Israil Israilevič, geb. 1905, parteilos, Jude, Halbanalphabet, in der Partisanenabteilung ab Februar 1943, geboren und ständig wohnhaft in der Ortschaft Naliboki, gemäß einiger Aussagen wegen Diebstahls und Brandstiftung vorbestraft, geprüft und 


\section{befunden:}

Anhand der Materialien des Falls und Zeugenaussagen ist festgestellt worden, daß I. I. Kesler zur Zeit der polnischen Macht in Naliboki Diebstähle, Brandstiftungen beging und ein Bordell unterhielt. Wegen Diebstahls und Brandstiftung wurde er wiederholt zu mehreren Jahren Freiheitsentzug verurteilt. Nach der Entlassung arbeitete er zugunsten der polnischen Abwehr (Defenzywa) und lieferte der polnischen Polizei Personen, die illegaler kommunistischer Tätigkeit verdächtigt wurden, aus. So lieferte er einen gewissen Štejn, ein aktives Mitglied der illegalen kommunistischen Partei, und mehrere andere an die polnische Polizei aus.

Als Westbelorußland durch die RKKA befreit wurde, flüchtete Kesler aus Angst vor der strafenden Hand der sowjetischen Justiz nach Litauen und kehrte nach Naliboki erst zurück, als die deutschen Besatzer das Territorium Westbelorußlands okkupierten, weil er glaubte, daß die deutschen Faschisten ihn wegen seiner früheren antikommunistischen Tätigkeit verschonen würden. Während der deutschen Okkupation lieferte er der deutschen Gendarmerie eine ganze Reihe ehemaliger sowjetischer Aktivisten und aktiver Komsomolzen aus, darunter die Partisanen unserer Abteilung Chonja Primakov und Abram Vajner, die jedoch der deutschen Polizei entgehen konnten.

1942 befand sich Kesler in der Ortschaft Dvorec, Rajon Novogrudok, von wo er jedoch floh, als die Deutschen im Dezember 1942 die ganze jüdische Bevölkerung von Dvorec vernichteten. In die Abteilung trat er im Februar 1943 ein.

In der Abteilung versuchte I. I. Kesler eine Zeitlang, aktiv für die Abteilung zu arbeiten, nahm an Beschaffungsoperationen teil, begann aber darauf wieder mit dem Marodieren und verprügelte die von ihm ausgeplünderten Bauern, ohne all die Verwarnungen der Führung zu beachten. Als er z.B. am 13.1.44 eine Beschaffungsaktion durchführte, nahm er den Abgebrannten von Naliboki die letzten Kühe und eine ganze Reihe von Sachen gesetzwidrig weg. In diesem Fall ging eine Beschwerde des Kommandeurs der Žukov-Brigade Major Vasilevič ein, der den Fall untersuchte und Marodieren und Banditentum feststellte. Durch sein Verhalten brachte Kesler die Partisanenbewegung bei der Bevölkerung in Mißkredit und schadete dadurch insbesondere unserer Partisanenabteilung.

In letzter Zeit bemühte sich Kesler, in der Abteilung die Autorität der Führung zu untergraben, verhinderte zu diesem $Z$ weck einige Maßnahmen und streute falsche Gerüchte bezüglich einiger Personen aus, um eine defätistische Stimmung in der Abteilung zu verbreiten und die Geschlossenheit von Abteilung und Führung zu schwächen.

Als Gruppenältester der Reservebasis schickte er trotz wiederholter Verwarnungen Leute auf eigene Faust, ohne Erlaubnis des Stabs, aus, damit sie auf den Gehöften Sachen gegen Schnaps und andere Lebensmittel für Kesler persönlich tauschten.

Am 2. 2. 44 brach er ohne Wissen des Stabs in unbekannter Richtung auf und verweigerte nach seiner Rückkehr am 4. 2. 44 jede Aussage.

Aufgrund des oben Dargelegten und in Anbetracht aller Umstände des Falls haben wir

beschlossen:

1. Kesler, Israil Israilevič, als ein antisowjetisches sozial gefährliches Element, einen Marodeur und Banditen zur Todesstrafe durch Erschießen zu verurteilen.

2. Den vorliegenden Beschluß als Befehl für die Abteilung abzufassen. 
3. Den vorliegenden Beschluß zusammen mit allen Materialien des Falls dem Bevollmächtigten der Sonderabteilung der Abteilung zur Aufbewahrung zu übergeben.

Abteilungskommandeur

i. A. Stellvertreter des Kommandeurs für politische Arbeit Stabschef

(Bel'skij)

(Gordon)

(Mal'bin)

F. 3623, op. 1, d. 2, 11. 107-108.

\section{Dokument Nr. 12}

\section{Meldung des Kommandeurs der Aufklärungsgruppe der Ponomarenko- Abteilung über die Entwaffnung der Partisanenabteilung Bel'skij}

(19. Februar 1944)

An den Kommandeur der Ponomarenko-Brigade

Major Ganzenko

von Gasjuk, Kommandeur der Aufklärungsgruppe

der Ponomarenko-Abteilung

\section{Meldung}

Ich, Kommandeur der Aufklärungsgruppe der Ponomarenko-Abteilung, habe dem Brigadekommando zu melden, daß ich eine Gruppe von Partisanen aus der Abteilung Bel'skij entwaffnet habe. Um 17.00 Uhr des 19. 2. 44 betrat eine Gruppe von Partisanen der Abteilung Bel'skij das Gehöft von Tarasov und öffnete unerlaubt die Kartoffelmiete, die dem Bauern Doščečko gehört. Sie füllten 20 Säcke mit Kartoffeln und wollten sich schon entfernen. In diesem Moment kamen ich und der Adjutant des Abteilungskommandeurs Sergeev an. Ich wies den Kommandeur der Gruppe Bel'skij darauf hin, daß auf den Gehöften von Tarasov ohne Erlaubnis nichts beschlagnahmt werden darf. Auf meinen Hinweis bekam ich eine grobe Antwort, man sagte mir, es gehe mich nichts an, ich solle mich nicht einmischen. Ich befahl zum zweiten Mal, die Kartoffeln zurückzugeben, worauf der Kommandeur der Gruppe Bel'skij seiner Gruppe befahl, sich zu zerstreuen, und sie schrien, ich sollte von ihren Fuhren weggehen. Ich gab drei Signalschüsse ab, worauf eine Gruppe von Kämpfern der Vasilevskij-Abteilung herbeiritt. Der Kommandeur der Gruppe der Vasilevskij-Abteilung befahl dem Kommandeur der Gruppe von Bel'skij, die Waffen abzuliefern. Sie befolgten den Befehl nicht, und wir mußten Gewalt anwenden.

Außerdem melde ich, daß, als ihr Kommandeur Litavodskij ihnen befahl, sich zu zerstreuen und liegen zu bleiben, auf mich geschossen wurde; die Kugel flog ganz nahe vorbei. Ich warte auf Ihren Befehl. An wen soll ich die Waffen übergeben? An den Stab der Abteilung Bel'skij oder den Brigadestab? Die Kartoffeln habe ich dem Bauern zurückgegeben. Warte auf Ihre weiteren Anordnungen.

Kommandeur der Aufklärungsgruppe

(Gasjuk)

F. 3623, op. 1, d. 1, 1. 80 . 


\section{Dokument Nr. 13}

\section{Meldung A. Bel'skijs über den Konflikt mit den Partisanen der Brigade „Vpered“ (29. Februar 1944)}

An den Gehilfen des Bevollmächtigten

des Stabes der Partisanenbewegung beim Hauptquartier

des Oberkommandos und des $\mathrm{ZK}$ der $\mathrm{KP}(\mathrm{b}) \mathrm{B}$

für die Oblast' Baranoviči

Gen. Sokolov

Lida-Raum

Kopie an

den Kommandeur der Brigade „Vpered“

Oberleutnant Bulat

\section{Meldung}

Am 29. Februar 1944 ritt ich in Begleitung des Kommandeurs einer Kampfgruppe und von Aufklärern, insgesamt $15 \mathrm{Mann}$, in unseren Operationsraum aus, um einen Kampfauftrag auszuführen und zwei Kompanien, die ich schon früher in den besagten Raum vorgeschickt hatte, zu leiten.

Nach dem Überqueren des Flusses kamen wir ins Dorf Malaja Ča[...]. Ich betrat mit einer Gruppe von Reitern das Haus des Bürgers Ivan Kozec, um auf den unterwegs zurückgebliebenen Schlitten, der uns nachfuhr, zu warten. Im Haus bat einer der Aufklärer die Hausfrau um ein Stück Brot. Da ich mich nach meiner Krankheit noch nicht sehr gut fühlte, legte ich mich aufs Bett und schlief kurz ein. Die Hausfrau gab kein Brot her, behauptete, keines zu haben, als aber ein Aufklärer auf einen Laib Brot wies, der auf einem Regal lag, griff sie nach dem Brot, versteckte es und schrie, sie hätte schon viel Brot gegeben, aber noch nichts dafür bekommen. („Ich habe mit euch Juden lange genug gelebt, ich kenne euch nur zu gut“; „Es reicht mir; ihr verfluchten Juden seid skrupellose Blutsauger.") Darauf sagte ihr der Kommandeur der Gruppe streng, vor ihr stünden nicht „verfluchte Juden“, sondern Partisanen. Sie schrie noch lauter und schimpfte, und in diesem Moment schlug er auf sie ein und stieß sie von sich weg. Die Hausfrau erhob ein Geschrei, ich stand vom Bett auf, schob den Kommandeur der Kampfgruppe zur Seite, beruhigte alle Anwesenden und befahl, eine Meldung über alles Vorgefallene zu schreiben. Darauf befahl ich, sich zum Abmarsch fertig zu machen, und schickte meine Leute zu den Pferden hinaus, blieb aber selber noch im Hause. In diesem Moment kam ein Partisan auf das Haus $\mathrm{zu}$, in der einen Hand hatte er eine Maschinenpistole, in der anderen einen dünnen Stock, er schwankte beim Gehen, es war zu sehen, daß er betrunken war. Er näherte sich den Leuten, die vor dem Haus standen, und fragte: „Wer hat die Hausfrau geschlagen?", und ein Junge wies auf den Kommandeur der Kampfgruppe: „Dieser da." Darauf kam er auf ihn zu und schlug ihm mit dem Stock ins Gesicht. Da liefen unsere Aufklärer herbei, sahen, wie der Betrunkene ihren Kommandeur verprügelte, und führten ihn sofort weg, um keine Prügelei zuzulassen, versuchten auch, ihm die Waffe wegzunehmen. In diesem Augenblick trat ich aus dem Hause und erkannte in dem Partisanen, der den Kommandeur der Kampfgruppe geprügelt hatte, meinen alten Bekannten, den Kommandeur der Čkalov-Abteilung Zajcev, 
wieder. Ich sagte meinen Leuten, sie sollten von ihm ablassen, und kam auf Zajcev zu, um mit ihm freundschaftlich zu reden. Er aber [...] beschimpfte mich grob, entsicherte seine Maschinenpistole und richtete sie gegen mich, wobei er sagte: „Sag, Bel'skij, weshalb hast du mich geprügelt?". Dann schrie er [...], er werde die Leute aus der Čkalov-, der „Sibirjak“- und der „Bol”ševik“-Abteilung zusammenrufen, schoß aus der MPi, gab zwei Salven in die Erde ab, beim dritten Mal schoß er in meine Richtung, die Kugel durchdrang meinen Gürtel und meinen Mantel. Ich war die ganze Zeit bemüht, ihn zu beschwichtigen, das gelang mir jedoch nicht. Er schimpfte fortwährend und schlug mir dann mit der MPi ins Gesicht, so daß ich schließlich blutüberströmt dastand. Da aber rannten weitere Partisanen mit dem Kommandeur der „Sibirjak“-Abteilung herbei, auch der Kompaniechef der „Bol’ševik“-Abteilung Denisov, der Kompaniechef V. Alanin aus der „Bol’ševik“-Abteilung und andere. Gen. Zajcev ließ zu, daß der Kommandeur alle entwaffnete.

Wieder wollte ich ihn beruhigen und schlug ihm selbst vor, meine MPi zu nehmen. Ich sagte auch all meinen Leuten, sie sollten die Waffen abgeben, um unnötiges Blutvergießen zu vermeiden. Die herbeigekommenen Partisanen fielen über meine Leute wütend her, verprügelten sie, nahmen ihnen die Waffen und die Munition weg. Ein Schuß fiel, dabei wurde die Feldbluse des Aufklärers Abramovič durchschossen. Der Oberaufklärer Rul'kovič, die Aufklärer Berkovič, Lejbovič und Bljacher mußten Prügel über sich ergehen lassen. Nachdem uns die Waffen weggenommen worden waren, trieb man uns alle ins Haus hinein, dort wurde ich weiter beschimpft, man nannte mich einen „Ataman“ und gebrauchte andere ähnliche Wörter. Zum Schluß drohten sie mir, sie werden mich noch in einen Hinterhalt locken, und gingen.

Während dieses Vorkommnisses ritten zwei von meinen Leuten, da sie die Gefährlichkeit der Situation sahen, zum den Stab der Brigade „Vpered“. Der Bevollmächtigte des Lida-Raums war gerade bei der Abteilung, auch der Kommandeur der Brigade „Vpered“ fehlte. Die Sache wurde dem Stabschef der Brigade gemeldet, der zusammen mit dem Chef der Sonderabteilung der Brigade zum Ort des Vorkommnisses kommen sollte. Inzwischen sammelte ich meine Leute, und wir begaben uns auf Umwegen ins Rajonzentrum. Im Rajonzentrum und im Brigadestab wurde befohlen, uns die Waffen zurückzugeben. Ich erhielt die Waffen aus der Abteilung "Sibirjak“, aber es fehlten folgende der weggenommenen Sachen:

1. ein Patronenstreifen für eine WIS-Pistole;

2. vier Trommelmagazine für Degtjarev-MPis;

3. eine Schlagfeder zu dieser MPi;

4. vier Revolver;

5. 195 Gewehrpatronen;

6. zwei Handgranaten;

7. drei Sättel;

8. zwei Zuführungseinrichtungen für Gewehre wurden durch untaugliche ersetzt.

Es fehlten auch andere Dinge, z.B. ein Ladestock für einen Zehnlader, ein Gewehrgurt usw. Der Kommandeur der Brigade „Vpered“ versprach, die oben genannten Waffen und die Munition bis zum 6. März d. J. zurückzugeben.

Die Kämpfer Boris Gul'kovič, Benjamin Berkovič und Il'ja Bljacher wurden bei der Prügelei verwundet. Was mich betrifft, so muß ich das Bett hüten. 
Gen. Bevollmächtigter, ich ersuche Sie:

1. zu verfügen, daß eine sorgfältige Untersuchung dieses Falls durchgeführt wird und die Schuldigen bestraft werden;

2. daß die uns weggenommenen Waffen, die Munition und die Sattel zurückgegeben werden. Ich meinerseits erteilte dem Kommandeur der Kampfgruppe eine strenge Rüge und gab ihm, damit er sich bewähre, den Kampfauftrag, im März zwei Züge zum Entgleisen zu bringen, auf der Chaussee eine Detonation auszulösen, die Nachrichtenverbindung abzuschneiden und mehrere Brücken längs der Straße in Brand zu stecken.

Expl. Nr. 1 für den Lida-Raum.

Nr. 2 an den Kommandeur der Brigade „Vpered“.

Nr. 3 zu den Akten.

Anlage: 2 Bescheinigungen.

4. 03.1944.

Nr. 0024.

\section{Bescheinigung}

Ausgestellt für den Kommandeur der Partisanenabteilung Bel'skij, Anatolij Davidovič, als Bescheinigung darüber, daß bei der medizinischen Untersuchung vom 1. 03. 44 folgendes festgestellt wurde:

1. ein subkutanes Hämatom und eine bedeutende Schwellung auf dem Rücken, an der linken Seite unterhalb der Rippen;

2. eine Wunde an der rechten Seite der Nase, $1 \mathrm{~cm}$ lang und $3 \mathrm{~mm}$ tief;

3. eine Wunde längs des rechten Kiefers, $5 \mathrm{~cm}$ lang und $5 \mathrm{~mm}$ tief.

Die oben genannten Körperverletzungen rühren von Schlägen mit einem stumpfen Gegenstand her und sind als mittelschwere Körperverletzungen einzustufen.

(Arzt Girš)

(Arzt Sinuk)

\section{Bescheinigung}

Ausgestellt an Gul'kovič, Boris Solomonovič, Oberaufklärer der Partisanenabteilung Bel'skij, als Bescheinigung darüber, daß bei der medizinischen Untersuchung vom 2. 03.1944 Folgendes festgestellt wurde:

Eine Wunde nahe der Braue des linken Auges, $3 \mathrm{~cm}$ lang, $2 \mathrm{~cm}$ breit, $1 \mathrm{~cm}$ tief.

Außerdem ein Bluterguß im Augenlid.

Die oben genannten Körperverletzungen rühren von Schlägen mit einem stumpfen Gegenstand her und sind als mittelschwere Körperverletzungen einzustufen. 


\section{Dokument Nr. 14}

\section{Angaben über die Zusammensetzung der Partisanenabteilung Bel'skij, Stand} per 1. April 1944

Formular Nr. 4

Streng gebeim

Angaben über die Zusammensetzung der Partisanenabteilung Bel'skij, Stand vom 1. April 1944

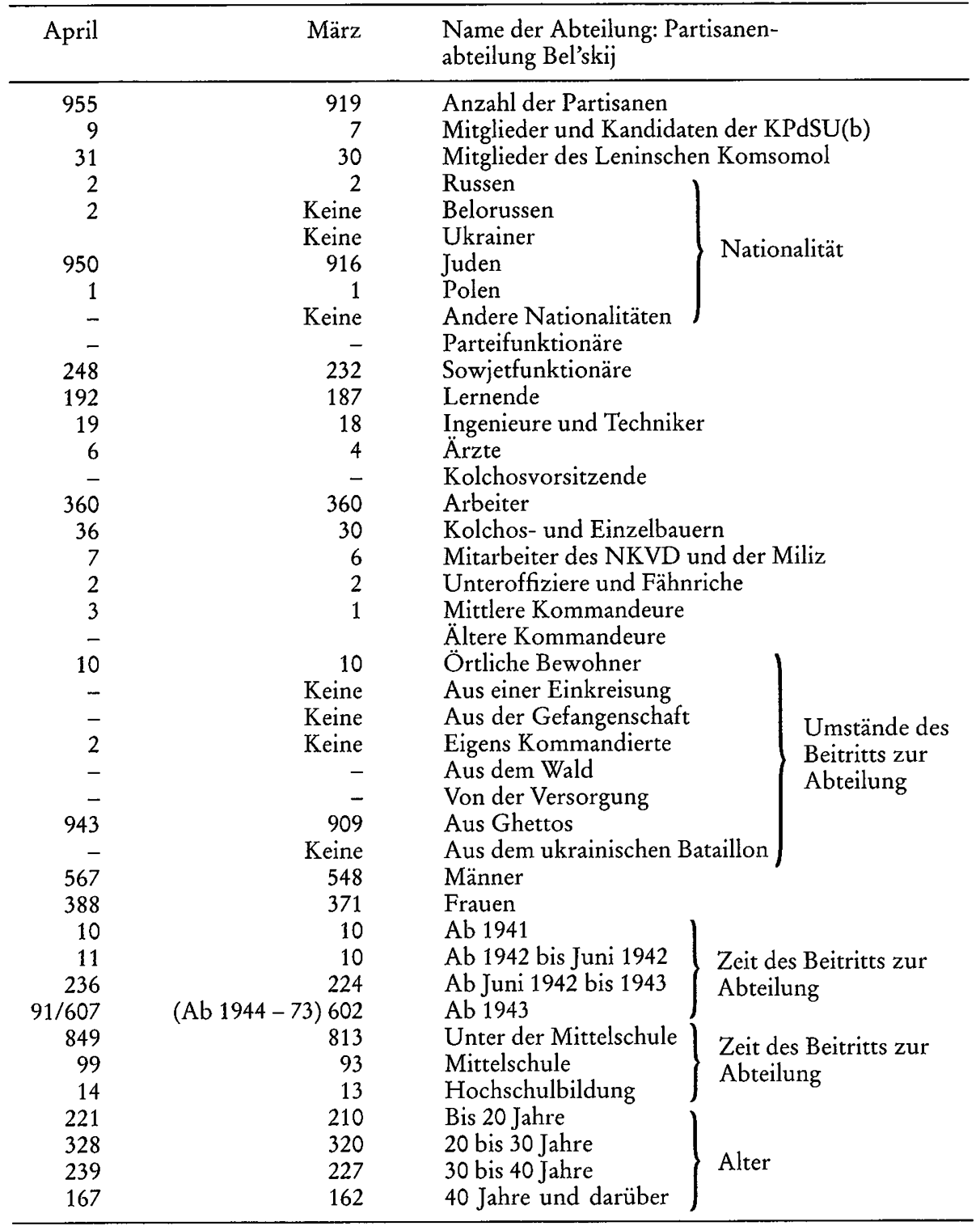




\begin{tabular}{ccl}
\hline April & März & $\begin{array}{l}\text { Name der Abteilung: Partisanen- } \\
\text { abteilung Bel'skij }\end{array}$ \\
\hline 26 & 25 & $\begin{array}{l}\text { Kinder bis 7 Jahre } \\
\text { in der Abteilung }\end{array}$ \\
98 & 96 & \begin{tabular}{l} 
Bis 16 Jahre \\
\hline
\end{tabular} \\
\hline
\end{tabular}

F. 3623, op. 1, d. 4, 1.21

\title{
Dokument Nr. 15
}

Tagesbefehlsbuch der Familiengruppe der Ordžonikidze-Partisanenabteilung und der Kalinin-Partisanenabteilung (6. Oktober 1943 - 12. Juli 1944) (Auszug)

\author{
Befehl \\ für die Familiengruppe der Ordžonikidze-Partisanenabteilung \\ $N r .02$
}

Wald ...

Zwecks Regelung des Lebens im Lager und Einrichtung des Lagers:

1. setze ich das Wecken auf $8 \mathrm{Uhr}$ morgens und den Zapfenstreich auf $22 \mathrm{Uhr}$ fest;

2. ernenne ich Gen. Potašnik, Maks Ošerovič, zum Kommandanten des Zentralen Baulagers; zu seinen Pflichten gehört folgendes:

A) für die Aufrechterhaltung der vollständigen Ordnung im Lager zu sorgen;

B) die Einhaltung des Weckens und des Zapfenstreichs im Lager zu überwachen;

C) auf die Aufrechterhaltung der Sauberkeit im Lager zu achten. $\mathrm{Zu}$ diesem Zweck wird Gen. Rubinštejn zum Sanitätsinspekteur ernannt und ihm mit seinem Personal als Hilfe beigegeben;

D) die Pferde, die sich im Lager befinden, im Lager eintreffen und das Lager verlassen, zu überwachen. Zu diesem Zweck wird ihm der Oberpferdehalter B. Kravec mit seinem Personal als Hilfe beigegeben;

E) auf die Ordnung in den Erdhütten zu achten; zu diesem Zweck werden ihm die Kommandanten der Erdhütten als Hilfe beigegeben, die die ganze Verantwortung für die Sauberkeit und Ordnung sowohl in als auch in der Umgebung der Erdhütten tragen;

F) die Wachen, getarnte Posten und die Diensthabenden im Lager zu überwachen;

G) auf Personen zu achten, die ins Lager kommen und es verlassen, ihnen gemeinsam mit dem Bauchef Übernachtungsstellen zuzuweisen, für ihre Verpflegung und die Furagierung ihrer Pferde zu sorgen;

$\mathrm{H})$ auf alle Leute zu achten, die nicht in Brigaden eingeteilt und nicht mit ihrer ständigen Arbeit beschäftigt sind.

3. Zu Punkt C 1):

Zum Sanitätsinspekteur des Zentralen Lagers wird Gen. Isaak Rubinštejn ernannt. Ihm wird eine fünf Mann starke Einheit beigegeben. Zu seinen Obliegenheiten gehört es, für die Einhaltung der vollständigen sanitären Sauberkeit im Lager zu 
sorgen, für die er die ganze Verantwortung trägt. Der Sanitätsinspekteur ist unmittelbar dem Lagerkommissar unterstellt.

4. Zu Punkt D 1):

Zum Oberpferdehalter des Zentralen Lagers wird Gen. Berko Kravec ernannt. Ihm wird ein Mitarbeiterstab von 10 Personen zugeordnet. Zu seinen Obliegenheiten gehört die Pflege und die richtige und vollständige Furagierung aller auf der Basis befindlichen Pferde. Der Oberpferdehalter ist unmittelbar dem Lagerkommandanten unterstellt.

5. Es ist streng verboten, sich müßig in den Abteilungswerkstätten aufzuhalten. Die Verantwortung für die Ordnung und die Ausführung der Aufträge wird dem technischen Leiter der Werkstätten Gen. Šmujlovič auferlegt.

6. Ich beauftrage Gen. M. Kabak zur Arbeit in den Werkstätten zur Annahme bei den Werkstätten eingehender Aufträge. Jeder Auftrag ist zur Kontrolle ins Auftragsbuch einzutragen und zu numerieren.

7. Zum Versorgungslagerleiter wird Gen. Lejba Abramovič ernannt, der aufgefordert wird, sofort das Lebensmittelgeschäft zu übernehmen und es vollständig in Ordnung zu bringen. Er hat ein Einnahmen- und Ausgabenheft für die Lebensmittel anzulegen. $\mathrm{Zu}$ seinem Stellvertreter wird der Bäckereileiter Gen. M. Geršovič ernannt.

8. Alle zum ständigen Aufenthalt auf der zentralen Basis eintreffenden Partisanen müssen sich unverzüglich im Abteilungsstab eintragen lassen. Wer sich nicht eingetragen hat, darf sich auf der Basis nicht länger als 24 Stunden aufhalten. Alle nicht angemeldeten Personen erhalten auf der Basis keine Verpflegung.

9. Alle sich im Lager aufhaltenden Partisanen werden gemäß den festgelegten Normen verpflegt. Brot erhalten sie ausschließlich vom Leiter des Verpflegungsdienstes Gen. Rozengauz. Er hat auch die Liste der Brotrationen.

10. Zum Verantwortlichen für die volle Auslastung der Abteilungsmühle ernenne ich Gen. Rezik, dem ein Mitarbeiterstab von 8 Personen zugeteilt wird.

11. Zum Verantwortlichen für die Fleisch- und Milchfarm wird Gen. Ch. Arman ernannt. Er trägt die volle Verantwortung für die Fütterung der Kühe und die Milcherträge. Er ist unmittelbar dem Lagerkommandanten unterstellt.

12. Die Pflichten des ständigen Kommandanten der Wache werden aufgehoben. Die Einteilung zum Wachdienst 24 Stunden vor Dienstbeginn vorgenommen. Wer zum Wachdienst ernannt ist, darf sich nicht aus dem Lager entfernen. Im Winter erfolgt die Ablösung von Wachen und Wachposten um $12 \mathrm{Uhr}$.

13. Die Wachen am Ein- und Ausgang des Zentralen Lagers dürfen Partisanen der eigenen Abteilung oder solche aus anderen Abteilungen ohne einen speziellen Passierschein ničht kommen und gehen lassen. Wenn Partisanen aus anderen Abteilungen das Lager betreten wollen, sind sie am Wachposten aufzuhalten und beim Abteilungsstab zu melden. Nach der Erlaubnis sind sie zum Stab zu bringen bzw. ist mit ihnen im Auftrag oder auf Weisung des Stabes zu verfahren. Die ganze Verantwortung für die Wachdienstdurchführung trägt der Wachhabende, der vom Stab alle 24 Stunden ernannt und abgelöst wird. Alle Fuhren, die ins Lager ein- oder aus dem Lager hinausfahren, müssen einen Passierschein haben.

14. Die Wache wird vor Dienstantritt täglich vom Abteilungsstab vollständig in ihre Aufgaben eingewiesen.

15. Streng verboten ist es, sich unmittelbar an mich als Abteilungskommandeur in 
welchen Fragen auch immer zu wenden. Das muß ausschließlich auf dem Dienstweg, über Unteroffiziere und Fähnriche erfolgen.

Kommandeur der Familiengruppe

bei der Ordžonikidze-Abteilung

Stabschef

\section{Befehl \\ für die Partisanenabteilung Bel'skij \\ $N r .001$}

Pušča N.

4. 1.44

1. Mit heutigem Datum wird die Familiengruppe bei der Ordžonikidze-Partisanenabteilung in die selbständige Partisanenabteilung Bel'skij eingegliedert.

Begründung: Befehl des Generalmajors Platon, Bevollmächtigter des Zentralstabes der Partisanenbewegung und des ZK der KP(b)B für die Oblast' Baranoviči

2. Zum Abteilungskommandeur bin ich ernannt worden.

3. Zum Stabschef wird Gen. L. A. Mal'bin ernannt.

4. Zum zeitweiligen Stellvertreter des Kommandeurs für politische Arbeit wird Gen. T. E. Gordon ernannt.

5. Den Stabschef weise ich an, unverzüglich alle Bewaffneten in der Abteilung zu ihrer Formierung zu einer Kampfgruppe zu erfassen. Die Einteilung der Gruppe in Kompanien, Züge und Gruppen sowie die Ernennung der Kommandeure wird durch einen speziellen Befehl festgelegt werden.

6. Ich weise alle Kämpfer der Abteilung an, alle Ersatzteile für jede Art von Waffen an den Stab abzuliefern. Termin: 7. 1. 44. Falls nach diesem Zeitpunkt bei den Kämpfern Ersatzteile festgestellt werden, sind die Betreffenden streng zu bestrafen.

7. Ich weise an, alle Überschüsse an Lebensmitteln, über die einzelne Personen verfügen, an das Lebensmittelgeschäft abzuliefern. Falls bei jemandem verdorbene Lebensmittel festgestellt werden, ist der Besitzer wegen Sabotage zur Verantwortung zu ziehen. Termin: 8. 1. 44.

8. Ich weise den Hauptfeldwebel Gen. Fridberg an, den Bau der Badeeinrichtung und der Entseuchungskammer zu beschleunigen.

9. Ich weise den Hauptfeldwebel Gen. Fridberg an, bis zum 10.1.44 Wachlokale zu bauen.

10. Ich weise den Sanitätsinspekteur Gen. Rubinštejn an, unverzüglich die Arbeit einzuleiten, um das Lager und die Erdhütten in den entsprechenden sanitären $\mathrm{Zu}$ stand zu bringen, und weise darauf hin, daß er bei antisanitärem Zustand des Lagers und der Erdhütten streng bestraft wird.

11. Der Partisanin Chiena Kalmanovič wird wegen des Streites mit dem Lagerkommandeur eine strenge Rüge erteilt.

12. Dem Partisanen Benjamin Dombrovskij erteile ich wegen seines Streites und Geschimpfes mit dem Lagerkommandanten eine strenge Rüge mit Verwarnung.

13. Alle Befehle und Anordnungen, die für die Familiengruppe der Ordžonikidze-Partisanenabteilung ausgegeben wurden, bleiben in Kraft.

Abteilungskommandeur

Stabschef

(Bel'skij)

i. A. Stellvertreter des Kommandeurs für politische Arbeit 


$$
\begin{gathered}
\text { Befebl } \\
\text { für die Partisanenabteilung Bel'skij } \\
\text { Nr. } 002
\end{gathered}
$$

Pušča N.

19. 01.44

1. Ich ernenne Rybak, Šlema Efimovič, zum Versorgungsleiter des Abteilungslazaretts.

2. Tareveckij, Bořs, ist wegen Nichtausführung einer Anordnung des Stabschefs mit verschärfter Haft von 5 Tagen zu bestrafen.

3. Abramovič, Leja Ovseevna, ist wegen unerlaubter Entfernung vom Posten mit verschärfter Haft von 5 Tagen zu bestrafen.

4. Boltaryskaja, Sara Geršovna, ist wegen falscher Aussagen gegenüber dem Bevollmächtigten des Rajonzentrums Stolbcy mit verschärfter Haft von 5 Tagen zu bestrafen.

5. Dviljanskij, Jakov Abramovič, ist wegen des von ihm begangenen Verbrechens - unerlaubtes Verlassen des Postens - zur Todesstrafe durch Erschießen zu verurteilen, aber mit Rücksicht auf sein früheres tadelloses Verhalten in der Abteilung ersetze ich die Erschießung durch verschärfte Haft von 5 Tagen, ab Tag der Befehlsausgabe.

6. Gorfunek, Boris, und Bernštejn, Boris, sind wegen Fernbleibens von der Arbeit ohne triftigen Grund mit geschäfter Haft von einem Tag zu bestrafen.

7. Wegen Vernachlässigung der Pflichten und Undiszipliniertheit erteile ich folgenden Partisanen eine strenge Rüge mit Verwarnung:

Bronickij, Meer,

Nejman,

Kopelevič, Samuil,

Novickij, Lejzer,

Rožanskij, Meer.

8. Die Ausführung des vorliegenden Befehls wird dem Lagerkommandanten auferlegt.
Abteilungskommandeur
(Bel'skij)
Stabschef
(Mal'bin)
i. A. Stellvertreter des Kommandeurs für politische Arbeit
(Gordon)

$$
\begin{gathered}
\text { Befebl } \\
\text { für die Partisanenabteilung Bel'skij } \\
N \text { r. } 006
\end{gathered}
$$

Pušča N.

1. 02.44

1. Mit heutigem Datum stelle ich eine Kampfgruppe von 180 Mann auf und ernenne:

Bel'skij, Asoël' Davidovič, zum Kommandeur der Kampfgruppe,

Abramovič, Ch. A., zum Chef der 1. Kompanie,

Ostašinskij, Ë. M., zum Führer des 1. Zuges der 1. Kompanie,

Cukerkon, M., zum Führer des 2. Zuges der 1. Kompanie,

Dombrovskij, B., zum Führer des 3. Zuges der 1. Kompanie,

Šušan, O. N., zum Führer des 4. Zuges der 1. Kompanie,

Novickij, L. V., zum zeitweiligen Chef der 2. Kompanie, 
Šlesberg, Ch. I., zum Führer des 1. Zuges der 2. Kompanie, Rybinskij, M. G., zum Führer des 2. Zuges der 2. Kompanie, Bel'skij, Ju. S., zum Führer des 3. Zuges der 2. Kompanie, Lejb, G., zum Führer des 4. Zuges der 2. Kompanie.

2. Der Stabschef hat zusammen mit dem Kommandeur der Kampfgruppe die Kämpfer in Kompanien und Züge einzuteilen. Nach der Einteilung sind die Listen mir zur Bestätigung vorzulegen.

Abteilungskommandeur

Stabschef

(Bel'skij)

i. A. Stellvertreter des Kommandeurs für politische Arbeit (Gordon)

\section{Befebl \\ für die Partisanenabteilung Bel'skij \\ Nr. 007}

Pušča N.

5. 02.44

1. Kesler, Izrail Izrail'evič, wird wegen Marodierens, Banditentums und wiederholten Ungehorsams zur Todesstrafe durch Erschießen verurteilt.

2. Das Urteil ist binnen 24 Stunden zu vollstrecken.

3. Der Befehl ist vor der Abteilung zu verlesen.

Abteilungskommandeur

(Bel'skij)

Stabschef

(Mal'bin)

i. A. Stellvertreter des Kommandeurs für politische Arbeit

(Gordon)

\section{Befebl \\ für die Partisanenabteilung Bel'skij \\ Nr. 0021}

Pušča N.

20.03. 44

1. In Anbetracht des klaren sommerlichen Wetters befehle ich, zur Sicherung des Lebens der im Lager befindlichen Menschen vor Angriffen der feindlichen Flugzeuge:

1. die Basis vollständig zu tarnen, damit aus der Luft keine Anzeichen zu erkennen sind;

2. mit Anbruch der Dunkelheit verstärkte Lichttarnung einzuführen.

3. Feuer zu machen ist von $8 \mathrm{Uhr}$ bis $19 \mathrm{Uhr}$ kategorisch verboten. In Ausnahmefällen ist Feuer, wenn es das Wetter erlaubt, mit Erlaubnis des Abteilungsstabes oder des Lagerkommandanten zu machen.

4. Personen, die gegen die Punkte des vorliegenden Befehls verstoßen, werde ich als Verräter bestrafen, bis hin zur Erschießung.

5. Die Ausführung des vorliegenden Befehls erlege ich dem Lagerkommandanten, den Wachhabenden im Lager, den Erdhüttenkommandanten und den Leitern der einzelnen Einheiten auf.

Abteilungskommandeur

Stabschef

(Bel'skij)

(Mal'bin)

i. A. Stellvertreter des Kommandeurs für politische Arbeit

(Gordon) 


\section{Befehl \\ für die Partisanenabteilung Bel'skij \\ Nr. 0023}

Pušča N.

26. 03.44

1. Tavrickij, Isaak Davidovič, ist wegen Nichtausführung der Befehle des Kompaniechefs und des Zugführers, des Fernbleibens von der Wache und unerlaubter Entfernung vom Wachposten ohne triftigen Grund zu entwaffnen und mit verschärfter Haft von 7 Tagen zu bestrafen sowie zu warnen, daß er beim nächsten Vergehen erschossen wird.

2. Černyj, Chaim Isaakovič, ist wegen Diebstahls (Stehlen von Speck bei einem Partisanen der Ordžonikidze-Abteilung) zu entwaffnen und mit geschäfter Haft von 7 Tagen zu bestrafen.

3. Pernikov, Izrail' Aronovič, ist wegen nachlässigen Umgangs mit einer fremden Waffe und der Abgabe eines versehentlichen Schusses, durch den der Partisan Bernak, Izrail', getötet wurde, mit verschärfter Haft von 5 Tagen zu bestrafen.

4. Chodak, Fajvel' Aronovič, ist wegen des Verlustes seines Gewehrs zur Todesstrafe durch Erschießen zu verurteilen, aber infolge seiner Minderjährigkeit ist die Höchststrafe durch verschärfte Haft von 10 Tagen zu ersetzen.

5. Gomberg, Ženja Ickovna, ist wegen unerlaubten Tausches des Wachdienstes mit Kac, Lejer, mit verschärfter Haft von 5 Tagen zu bestrafen.

6. Kac, Lejer, ist wegen unerlaubten Tausches des Wachdienstes mit Gomberg mit verschärfter Haft von 5 Tagen zu bestrafen.

Abteilungskommandeur

Stabschef

(Bel'skij)

i. A. Stellvertreter des Kommandeurs für politische Arbeit

(Gordon)

\section{Pušča N.}

An die Mannschaften

1. Für die Erfüllung der Gefechtsaufgabe der Leitung, die ihren Ausdruck darin fand, daß 2 gegnerische Züge zum Entgleisen gebracht, hierbei 2 Lokomotiven beschädigt, 13 Waggons vernichtet und 4 Waggons mit für die Front bestimmter Fracht beschädigt wurden, außerdem ein feindliches Kraftfahrzeug mit 12 Hitlerleuten vernichtet wurde, spreche ich folgenden Partisanen meiner Abteilung, die die Gefechtsaufgabe erfüllt haben, den Dank aus, der auch in die Personalakte eingetragen wird: den Genossen Cukerkop, Mejlach Pinchusovič; Feruman, L'ev Zel'manovič; Mitljanskij, David Moiseevič; Ostašinskij, Daniel Efimovič; Dvoreckij, Abram Pejsachovič; Šljachtovič, Abram Isaakovič; Rožanskij, Meer Abramovič; Zegerman, Jankel Efremovič; Abramovič, Iosif Abramovič; Mitivinskij, Abram Moiseevič; Janson, Wol'f Davidovič; Vygodskij, David Volf'ovič; Ljubčanskij, Aron Jankelevič; Olengejm, Ovsej Samujlovič; Berman, Jakov Ziselevič; Jatvickij, Lejzer Bencianovič; Novickij, Šišma Girševič; Novickij, Jakov Aronovič; Kagan, Marusja Iosifovna; Ochman, Maks Isaakovič. Die Abteilungsführung ist fest davon überzeugt, daß ihr 
auch künftig noch größere Leistungen aufweisen und dem Feind noch stärkere Schläge versetzen werdet.

2. Für die gewissenhafte Pflege der Verwundeten spreche ich den medizinischen Schwestern Libo, Tat'jana, und Švarc, Lilja, den Dank mit Eintragung in die Personalakte aus.

3. Der Befehl ist vor der angetretenen Abteilung zu verlesen.

Abteilungskommandeur

(Bel'skij)

Stabschef

Abteilungskommissar

(Mal'bin)

(Šemjatovec)

\section{Befebl \\ für die Partisanenabteilung Bel'skij \\ Nr. 0033}

Pušča N.

6. 05.44

1. Mit heutigem Datum wird die Partisanenabteilung Bel'skij in die Partisanenabteilung „M. I. Kalinin“ umbenannt.

Begründung: Befehl Nr. 011 des Bevollmächtigten des Stabes der Partisanenbewegung und des ZK der KP(b)B für die Oblast' Baranoviči, Rajon Lida, vom 4. 5. 44.

2. Zum stellvertretenden Kommissar der Kalinin-Abteilung für Komsomolarbeit wird Gen. Presman, Chonja Gercovič, ernannt. Begründung: Schreiben des Sekretärs des Oblastkomitees des Leninschen Komsomol vom 26. 04. 44.

3. Der Partisan Ljubčanski, Aron Janklevič, ist wegen Simulation und systematischer Weigerung, die Aufträge und Anordnungen der Leitung zu erfüllen, mit verschärfter Haft von 10 Tagen zu bestrafen.

4. In Anbetracht des Internationalen proletarischen Feiertages, des 1. Mai, gilt die Strafe für die Gen. Ljubčanskij, Aron Janklevič, Saganovič, Rachmiel' Il'jič, und Gurvič, Ven'jamin Isaakovič, als vorfristig abgebüßt.

Abteilungskommandeur
Stabschef
Abteilungskommissar
(Bel'skij)

(Mal'bin)

(Šemjatovec)

\section{Befebl \\ für die Kalinin-Partisanenabteilung \\ $N r .0039$}

\section{Pušča N.}

27. 06.44

1. Trotz wiederholter Einweisungen und Instruktionen bei der Entsendung einzelner Gruppen zu Operationen kommt es vor, daß Partisanen unserer Abteilung der direkten Ausführung des Auftrags der Leitung aus dem Wege gehen und Beschaffungsoperationen für ihre persönlichen Bedürfnisse durchführen. So traf die 5 Mann starke Gruppe (Gruppenleiter Lipnik, die Partisanen Lifšic, Gul'nik, Movšovič, Miranker), die ins Dorf Derevnoe entsandt wurde, um Baustoffe zu holen, unterwegs auf 2 Kühe. Sie trieben die Kühe zum Lager, schlachteten unterwegs aber eine davon und teilten das Fleisch unter sich. Die Partisanen Berkovič sowie Chejfec, Bojarskij und Šifmanovič, die nach technischen Materialien entsandt wur- 
den, gingen, statt den Auftrag rechtzeitig auszuführen, daran, im Dorf Lebensmittel für ihren persönlichen Bedarf zu beschaffen. Das Verhalten der oben genannten Partisanen untergräbt zweifellos die Maßnahmen der Abteilungsführung, trägt Zersetzung in die Partisanenreihen hinein und tut dem Ansehen unserer Abteilung in den Augen der anderen Abteilungen und der Bevölkerung eindeutig Abbruch. Diese verbrecherischen Handlungen verdienen die strengste Strafe, bis hin zur Erschießung. Das trifft besonders auf Personen zu, die diesbezüglich schon verwarnt wurden.

2. Aufgrund des oben Dargelegten befehle ich:

folgende Personen mit verschärfter Haft von 10 Tagen zu bestrafen:

1. Lipnik, Girš Jankelevič,

2. Gul'nik, Moisej Moiseevič,

3. Lifšic, Boris Lejbovič,

4. Miranker, Girš Evelevič,

5. Chejfec, Evel Šaevič,

6. Bojarskij, Tanchel' Fal'kovič.

3. Šifmanovič, Jankel' Jankelevič, ist mit verschärfter Haft von 5 Tagen zu bestrafen.

4. Berkovič, Moisej Aronovič, und Movšovič, Il'ja Samujlovič, ist eine strenge Rüge mit Verwarnung zu erteilen.

5. Wegen Verschleuderung des Abteilungsbesitzes, die im Diebstahl von Leder zu persönlichen Zwecken bestand, ist Gen. Kabak, Matus Ioselevič, mit verschärfter Haft von 10 Tagen zu bestrafen.

6. Wegen waffenlosen Erscheinens im Stab auf Aufforderung ist Gen. Kabak, M. I., mit verschärfter Haft von 3 Tagen zu bestrafen.

7. Gen. Kabak, M. I., ist insgesamt mit verschärfter Haft von 13 Tagen zu bestrafen, wobei ihm bis zu seiner Besserung das Recht, Waffen zu tragen, entzogen wird.

8. In Anbetracht des oben Dargelegten setze ich Gen. Kabak vom Posten des Leiters der Abteilungswerkstätten ab.

9. Zum Leiter der Abteilungswerkstätten ernenne ist Gen. Šljachtovič, Abram Isaakovič, ohne ihn vom Amt des Politruks der 1. Kompanie zu entpflichten.
Abteilungskommandeur
Stabschef
Abteilungskommissar
(Bel'skij)
(Mal'bin)
(Šemjatovec)

\section{Befebl \\ für die Kalinin-Partisanenabteilung \\ $N r .0040$}
Pušča N.
8. 07.44

1. Auf Anordnung des Oblastzentrums befehle ich: Es ist verboten, jegliche deutsche Uniformstücke, mit Ausnahme des Schuhwerks, zu tragen. Bei Nichterfüllung des vorliegenden Befehls werde ich die Schuldigen zu strengster Verantwortung ziehen.
Abteilungskommandeur
(Bel'skij)
Stabschef
Abteilungskommissar 


$$
\begin{gathered}
\text { Befebl } \\
\text { für die Kalinin-Partisanenabteilung } \\
\text { Nr. } 0041
\end{gathered}
$$

Stadt Novogrudok

12. 07.44

1. Infolge der Befreiung der Abteilung durch die Rote Arbeiter- und Bauernarmee gilt die Abteilung ab heute als aufgelöst.

2. Die gesamt Mannschaft hat sich zum jeweiligen ständigen Wohnort zu begeben, um sich dort beim entsprechenden Kriegskommissariat zu melden, wofür die üblichen Ausweise ausgegeben worden sind.

3. Der Stabschef hat die Papiere und Akten der Abteilung vorzubereiten, um sie dem Belorussischen Stab der Partisanenbewegung zu übergeben.
Abteilungskommandeur
Stabschef
Abteilungskommissar

\author{
(Bel'skij) \\ (Mal'bin) \\ (Šemjatovec)
}

F. 3623, op. 1, d. 3, 11. 1-47.

\title{
Dokument Nr. 16 \\ Kriegstagebuch der Kampfhandlungen der Abteilung Bel'skij \\ (4. Februar - 22. Juni 1944) \\ Kriegstagebuch der Kampfhandlungen der Abteilung Bel'skij
}

1. 13. 2. 44. Um 20.30 Uhr am 4. Februar 1944 auf der Strecke Baranoviči - Lida, in einer Entfernung von $2 \mathrm{~km}$ von der Station Jacuki beim Bahnübergang Guščanski, in Richtung auf die Station Neman, Zug zum Entgleisen gebracht; eine Lokomotive und 7 Waggons zerstört, 4 Waggons beschädigt. Zugverkehr für 15 Stunden aufgehalten.

2. 22. 3. 44. Um 17.00 Uhr am 17. März 1944 auf der Strecke Baranoviči - Lida, Abschnitt Neman - Jacuki, Zug mit Militärgut zum Entgleisen gebracht. Lokomotive und 6 Waggons zerstört. Zugverkehr um 9 Stunden aufgehalten.

3. 28. 3. Um 12.00 am 22. März auf der Chaussee Novogrudok - Lida, in einer Entfernung von $6 \mathrm{~km}$ von der Station Neman an dem Fluß Černaja, Kraftfahrzeug gesprengt. Kraftfahrzeug vernichtet, Brïcke beschädigt, 12 Hitlerleute getötet.

4. 30. 3. In der Nacht vom 26. zum 27. März 1944 Fernsprech- und Telegrafieverbindung an der Strecke Baranoviči - Lida, Abschnitt Novoel'naja - Dvorec, auf einer Länge von 1 (einem) Kilometer (21 Maste) vernichtet. Beim Rückzug ein Gefecht mit Polizeihinterhalt. Ein Polizist getötet, eine Magazintasche und 100 Patronen für russisches Gewehr erbeutet.

5. 16. 4. Um 3.00 Uhr Moskauer Zeit am 11. April 1944 an der Strecke Molodečno - Lida, Abschnitt Wajgony - Jachimovščina, Zug mit Rüstungsgütern zum Entgleisen gebracht. Lokomotive und 4 Waggons beschädigt. Zugverkehr auf der Strecke für 5 Stunden aufgehalten. TNT-Verbrauch: $8 \mathrm{~kg}$.

6. 27. 04. Am 18. April 1944 die Bürger Januško, Michail, und Smoljak, Pavel, im 
Dorf Staryj Les, Rajon Novogrudok, von einer Partisanengruppe der Abteilung Bel'skij gefangen und nach der Identifikation als Personen, die in der Belorussischen Armee von Ragulja dienten, erschossen.

7. 30. 04. Am 27. April 1944 auf der Chaussee Novogrudok - Lida, in der Nähe des Dorfes Rusilovka, Rajon Novogrudok, von einem Zug der Reiteraufklärungsabteilung mittels zweier TNT-Minen 2 deutsche Kraftfahrzeuge gesprengt, 3 Hitlerleute und 2 Polizisten getötet. TNT-Verbrauch: $8 \mathrm{~kg}$.

8. 30. 04. Um 4 Uhr am 28. April 1944 auf der Strecke Baranoviči - Lida, Abschnitt Neman - Jacuki, Zug des Gegners zum Entgleisen gebracht, TNT-Verbrauch: $8 \mathrm{~kg}$. Lokomotive beschädigt, 4 Waggons mit Pontonbrücken zerstört. Verkehr für 9 Stunden aufgehalten. Sprengung durch einen Zug der Reiteraufklärungsabteilung vorgenommen.

9. 30. 04. Am 29. April 1944 auf der Chaussee Novogrudok - Lida beim Dorf Orkoviči durch den Zug der Reiteraufklärungsabteilung deutsches Kraftfahrzeug gesprengt. Ein Hitlermann und 2 Polizisten getötet. TNT-Verbrauch: 4 kg.

10. 30. 04. Um 9 Uhr morgens am 29. April 1944 auf der Chaussee Novogrudok - Lida beim Gehöft Sacharovščina durch einen Sprengtrupp unter der Leitung von Ponimovskij deutsches Kraftfahrzeug gesprengt. 2 Polizisten getötet, einer verwundet. TNT-Verbrauch: $4 \mathrm{~kg}$.

11. 30. 04. Um 16 Uhr am 29. April 1944 auf der Chaussee Novogrudok - Lida durch einen Sprengtrupp unter Leitung von Ponimovskij deutsches Kraftfahrzeug mit Mannschaften (genaue Zahl nicht festgestellt) gesprengt und verbrannt. TNTVerbrauch: $5 \mathrm{~kg}$. Sprengung neben der Brücke beim Dorf Mitkeviči vorgenommen.

12. 20. 05. Um 20 Uhr am 18. Mai 1944 zog sich ein Zug der Reiteraufklärungsabteilung unter der Leitung des Kommandeurs der Kampfgruppe Bel'skij und des Stabschefs Mal'bin, L. N., auf Anordnung des Brigadekommandeurs Gen. Kudrin kämpfend von den Atminovskie-Gehöften zurück und legte beim Dorf Kriviči einen Hinterhalt an. Der Zug beschoß eine sich nähernde Gruppe von Weißpolen und wich danach zurück. Nach Mitteilungen 6 Mann getötet; Zahl der Verwundeten nicht festgestellt.

13. 20. 05. Um 14 Uhr am 12. Mai 1944 auf der Chaussee Novogrudok - Lida, $17 \mathrm{~km}$ von Novogrudok und $4 \mathrm{~km}$ von Novaja Guta entfernt, deutscher Pkw gesprengt. 4 Hitlerleute getötet. Gruppenleiter: Astašinskij. TNT-Verbrauch: $5 \mathrm{~kg}$.

14. 30. 05. Am 19. Mai 1944 wurde eine 21 Mann starke Partisanengruppe bei der Rückkehr von einer Versorgungsoperation im Dorf Podkosov'e von der Polizei aus der Garnison in Nečneviči beschossen. Die Gruppe wich unter Kämpfen, wobei der Politruk der 1. Kompanie Šljachtovič, wie von der Aufklärung der Kotovskij-Abteilung festgestellt, einen Polizisten schwer verwundete.

15. 6. 06. Um 10 Uhr des 27. Mai 1944 auf der Chaussee Novogrudok - Lida, Abschnitt Litovka - Novogrudok, durch einen Zug der Reiteraufklärungsabteilung 2 deutsche Kraftfahrzeuge gesprengt, 6 Hitlerleute getötet, 4 verwundet. TNT-Verbrauch: $8 \mathrm{~kg}$.

16. 6. 06. Um 9 Uhr 29. Mai 1944 auf der Chaussee Novogrudok - Lida, Abschnitt Dorf Ostrovnja - Guta, durch einen Zug der Reiteraufklärungsabteilung 2 deutsche Kraftfahrzeuge gesprengt, eines davon, mit Mannschaften, deren Zahl nicht festgestellt wurde, verbrannt. Im zweiten $\mathrm{Kfz} 2$ Hitlerleute getötet, 1 schwer verwundet. TNT-Verbrauch: $8 \mathrm{~kg}$. 
17. 6. 06. Um 10 Uhr am 1. Juni 1944 auf der Chaussee Novogrudok - Lida, am Fluß Černaja bei Staraja Guta, durch einen Zug der Reiteraufklärungsabteilung Holzbrücke gesprengt. Verkehr für 2 Tage unterbrochen. TNT-Verbrauch: $4 \mathrm{~kg}$.

18. 6. 06. Am 4. Juni 1944 auf der Chaussee Novogrudok - Wseljub, nahe des Dorfes Karnyši, durch einen Zug der Reiteraufklärungsabteilung 2 Kraftfahrzeuge gesprengt, 3 Hitlerleute, 3 Kosaken getötet, 4 schwer verwundet. TNT-Verbrauch: $8 \mathrm{~kg}$.

19. 7. 06. Um 13 Uhr am 4. Juni 1944 auf der Chaussee Voložin - Višnevo, Abschnitt Sokovščina - Slokovščina im Bombolovskij-Wald, durch einen Sprengtrupp unter Zugführer Janson, Viktor, gegnerisches Kraftfahrzeug mit Mannschaften gesprengt. Deren Zahl konnte die Aufklärung nicht feststellen.

20. 15. 06. Sprengtrupp unter Šuplan, Oter, zurück: Hohe Konzentration der Weißkosaken im Rajon verhinderte weitere Bewegung.

21. 22. 06. In der Nacht vom 14. zum 15. Juni Gleise bei der Station Gav'ja, Strecke Molodečno - Lida, durch einen Sprengtrupp unter Kukelko, Anton, vermint. Um 3 Uhr am 15.06 Zug zum Entgleisen gebracht, Lokomotive und 4 Lazarettwaggons beschädigt. Um 7 Uhr am 15. Juni von derselben Gruppe am selben Ort Zug mit Rüstungsgütern zum Entgleisen gebracht, Lokomotive und 5 Waggons beschädigt. TNT-Verbrauch für beide Sprengungen: $16 \mathrm{~kg}$.

F. 3623, op. 1, d. 5, 1l. 1-10. 